Chao Ji · Vicenţiu D. Rădulescu

\title{
Multi-bump solutions for the nonlinear magnetic Schrödinger equation with exponential critical growth in $\mathbb{R}^{2}$
}

Received: 29 November 2019 / Accepted: 18 March 2020 /

Published online: 30 March 2020

Abstract. In this paper, using variational methods, we establish the existence and multiplicity of multi-bump solutions for the following nonlinear magnetic Schrödinger equation

$$
-(\nabla+\mathrm{i} A(x))^{2} u+(\lambda V(x)+Z(x)) u=f\left(|u|^{2}\right) u \text { in } \mathbb{R}^{2},
$$

where $\lambda>0, f(t)$ is a continuous function with exponential critical growth, the magnetic potential $A: \mathbb{R}^{2} \rightarrow \mathbb{R}^{2}$ is in $L_{\text {loc }}^{2}\left(\mathbb{R}^{2}\right)$ and the potentials $V, Z: \mathbb{R}^{2} \rightarrow \mathbb{R}$ are continuous functions verifying some natural conditions. We show that if the zero set of the potential $V$ has several isolated connected components $\Omega_{1}, \ldots, \Omega_{k}$ such that the interior of $\Omega_{j}$ is non-empty and $\partial \Omega_{j}$ is smooth, then for $\lambda>0$ large enough, the equation has at least $2^{k}-1$ multi-bump solutions.

\section{Introduction and main results}

This paper is devoted to the qualitative analysis of solutions for the nonlinear magnetic Schrödinger equation in $\mathbb{R}^{2}$. We are concerned with the existence and multiplicity of multi-bump solutions if the reaction has an exponential critical behavior. In the first part of this section, we recall some significant historical moments related to the development of the Schrödinger theory. The main result and an associated multiplicity property are described in the second part of the present section.

\subsection{Historical comments}

The Schrödinger equation is central in quantum mechanics and it plays the role of Newton's laws and conservation of energy in classical mechanics, that is, it predicts

C. Ji: Department of Mathematics, East China University of Science and Technology, Shanghai 200237, China. e-mail: jichao@ecust.edu.cn

V. D. Rădulescu ( $\varangle)$ : Faculty of Applied Mathematics, AGH University of Science and Technology, 30-059 Kraków, Poland

Department of Mathematics, University of Craiova, 200585 Craiova, Romania

Nonlinear Analysis and Applied Mathematics (NAAM) Research Group, Department of Mathematics, Faculty of Science, King Abdulaziz University, P.O. Box 80203, Jeddah 21589, Saudi Arabia. e-mail: radulescu@inf.ucv.ro

Mathematics Subject Classification: 35J60 · 35Q55 · 35B33 
the future behaviour of a dynamical system. It is striking to point out that talking about his celebrating equation, Erwin Schrödinger said: "I don't like it, and I'm sorry I ever had anything to do with it". The linear Schrödinger equation is a central tool of quantum mechanics, which provides a thorough description of a particle in a non-relativistic setting. Schrödinger's linear equation is

$$
\Delta u+\frac{8 \pi^{2} m}{\hbar^{2}}(E-V(x)) u=0,
$$

where $u$ is the Schrödinger wave function, $m$ is the mass of the particle, $\hbar$ denotes Planck's renormalized constant, $E$ is the energy, and $V$ stands for the potential energy.

Schrödinger also established the classical derivation of his equation, based upon the analogy between mechanics and optics, and closer to de Broglie's ideas. He developed a perturbation method, inspired by the work of Lord Rayleigh in acoustics, proved the equivalence between his wave mechanics and Heisenberg's matrix, and introduced the time dependent Schrödinger's equation

$$
i \hbar u_{t}=-\frac{\hbar^{2}}{2 m} \Delta u+V(x) u-\gamma|u|^{p-1} u \quad x \in \mathbb{R}^{N}(N \geq 2),
$$

where $p<2 N /(N-2)$ if $N \geq 3$ and $p<+\infty$ if $N=2$.

In physical problems, a cubic nonlinearity corresponding to $p=3$ in Eq. (1.1) is common; in this case problem (1.1) is called the Gross-Pitaevskii equation. In the study of Eq. (1.1), Floer and Weinstein [24] and Oh [37] supposed that the potential $V$ is bounded and possesses a non-degenerate critical point at $x=0$. More precisely, it is assumed that $V$ belongs to the class $\left(V_{a}\right)$ (for some real number $a$ ) introduced in Kato [30]. Taking $\gamma>0$ and $\hbar>0$ sufficiently small and using a Lyapunov-Schmidt type reduction, Oh [37] proved the existence of bound state solutions of problem (1.1), that is, a solution of the form

$$
u(x, t)=e^{-i E t / \hbar} u(x) .
$$

Using the Ansatz (1.2), we reduce the nonlinear Schrödinger equation (1.1) to the semilinear elliptic equation

$$
-\frac{\hbar^{2}}{2 m} \Delta u+(V(x)-E) u=|u|^{p-1} u .
$$

The change of variable $y=\hbar^{-1} x$ (and replacing $y$ by $x$ ) yields

$$
-\Delta u+2 m\left(V_{\hbar}(x)-E\right) u=|u|^{p-1} u \quad x \in \mathbb{R}^{N},
$$

where $V_{\hbar}(x)=V(\hbar x)$.

Let us also recall that in his 1928 pioneering paper, Gamow [25] proved the tunneling effect, which lead to the construction of the electronic microscope and the correct study of the alpha radioactivity. The notion of "solution" used by him was not explicitly mentioned in the paper but it is coherent with the notion of weak solution introduced several years later by other authors such as J. Leray, L. Sobolev 
and L. Schwartz. Most of the study developed by Gamow was concerned with the bound states $u(x, t)$ defined in (1.2), where $u$ solves the stationary equation

$$
-\Delta u+V(x) u=\lambda u \text { in } \mathbb{R}^{N},
$$

for a given potential $V(x)$. Gamow was particularly interested in the Coulomb potential but he also proposed to replace the resulting potential by a simple potential that keeps the main properties of the original one. In this way, if $\Omega$ is a subdomain of $\mathbb{R}^{N}$, Gamow proposed to use the finite well potential

$$
V_{q, \Omega}(x)=\left\{\begin{array}{ll}
V(x) & \text { if } x \in \Omega \\
q & \text { if } x \in \mathbb{R}^{N} \backslash \Omega
\end{array} \quad \text { for some } q \in \mathbb{R} .\right.
$$

It seems that the first reference dealing with the limit case, the so-called infinite well potential,

$$
V_{\infty}\left(x ; R, V_{0}\right)=\left\{\begin{array}{l}
V_{0} \text { if } x \in \Omega \\
+\infty \text { if } x \in \mathbb{R}^{N} \backslash \Omega
\end{array} \quad \text { for some } V_{0} \in \mathbb{R},\right.
$$

was the book by the 1977 Nobel Prize Mott [36]. The more singular case in which $V_{0}$ is the Dirac mass $\delta_{0}$ is related with the so-called Quantum Dots, see Joglekar [29]. In contrast with classical mechanics, in quantum mechanics the incertitude appears (the Heisenberg principle). For instance, for a free particle (i.e. with $V(x) \equiv 0$ ), in nonrelativistic quantum mechanics, if the wave function $u(\cdot, t)$ at time $t=0$ vanishes outside some compact region $\bar{\Omega}$ then at an arbitrarily short time later the wave function is nonzero arbitrarily far away from the original region $\bar{\Omega}$. Thus, the wave function instantaneously spreads to infinity and the probability of finding the particle arbitrarily far away from the initial region is nonzero for all $t>0$. We refer to Díaz [20] for more details. Finally, we point out that sublinear Schrödinger equations with lack of compactness and indefinite potentials have been studied by Bahrouni, Ounaies and Rădulescu [10,11].

\subsection{Main results}

Consider the following nonlinear Schrödinger equation

$$
-\Delta u+(\lambda V(x)+Z(x)) u=f(u), \quad x \in \mathbb{R}^{N},
$$

where $\lambda>0$ is a parameter, $V, Z, f$ are continuous functions verifying some assumptions, has been studied by many researchers. In [21], Ding and Tanaka studied problem (1.4) assuming $f(t)=|t|^{q-1} t$. In this mentioned paper, the authors established the existence of multi-bump positive solutions for the problem

$$
\left\{\begin{array}{l}
-\Delta u+(\lambda V(x)+Z(x)) u=u^{p}, \quad x \in \mathbb{R}^{N}, \\
u \in H^{1}\left(\mathbb{R}^{N}\right),
\end{array}\right.
$$

where $2<p<2 N /(N-2)$ for $N \geq 3$ and $2<p<\infty$ for $N=1,2$. The authors showed that problem (1.5) has at least $2^{k}-1$ multi-bump solutions for $\lambda$ 
large enough. These solutions have the following properties: for each non-empty subset $\Gamma \subset\{1,2, \ldots, k\}$ and $\epsilon>0$ fixed, there is $\lambda^{*}>0$ such that problem (1.5) possesses a solution $u_{\lambda}$ for all $\lambda \geq \lambda^{*}=\lambda^{*}(\epsilon)$, satisfying:

$$
\left|\int_{\Omega_{j}}\left(\left|\nabla u_{\lambda}\right|^{2}+(\lambda V(x)+Z(x))\left|u_{\lambda}\right|^{2}\right) d x-\left(\frac{1}{2}-\frac{1}{p+1}\right)^{-1} c_{j}\right|<\epsilon, \forall j \in \Gamma
$$

and

$$
\int_{\mathbb{R}^{N} \backslash \Omega_{\Gamma}}\left(\left|\nabla u_{\lambda}\right|^{2}+(\lambda V(x)+Z(x))\left|u_{\lambda}\right|^{2}\right) d x<\epsilon,
$$

where $\Omega_{\Gamma}=\bigcup_{j \in \Gamma} \Omega_{j}$ and $c_{j}$ is the minimax level of the energy functional related to the problem

$$
\begin{cases}-\Delta u+Z(x) u=u^{p}, & \text { in } \Omega_{j}, \\ u>0, & \text { in } \Omega_{j}, \\ u=0, & \text { on } \partial \Omega_{j} .\end{cases}
$$

In [2], using variational methods, Alves et al. considered the existence of multibump positive solutions for the following problem with critical growth

$$
-\Delta u+(\lambda V(x)+Z(x)) u=\beta u^{p}+u^{2^{*}-1}, \quad x \in \mathbb{R}^{N},
$$

where $\lambda, \beta>0, p \in\left(1,2^{*}-1\right), 2^{*}=2 N / N-2, N \geq 3$. In [2], due to the critical growth of the nonlinearity in $\mathbb{R}^{N}$, the method applied in [21] does not hold. In [8], using a new approach, Alves et al. established the same results for the following equation

$$
-\Delta u+(\lambda V(x)+Z(x)) u=f(u), \quad x \in \mathbb{R}^{2},
$$

where $f$ is continuous with exponential critical growth. Due to the exponential critical growth of the nonlinearity in $\mathbb{R}^{2}$, some estimates in [8] are completely different from the case $N \geq 3$. For the further research about the nonlinear Schrödinger equation with the deepening potential well, we refer to $[1,4-7,9,12,27,34]$ and their references.

In recent years, the nonlinear magnetic Schrödinger equation

$$
i \hbar \frac{\partial u}{\partial t}=\left(\frac{\hbar}{i} \nabla-A(x)\right)^{2} u+U(x) u-f\left(|u|^{2}\right) u \text { in } \mathbb{R}^{N} \times \mathbb{R} .
$$

has also received considerable attention. This class of problems has some relevant physical applications, such as nonlinear optics and plasma physics. The function $u(x, t)$ takes on complex values, $\hbar$ is the Planck constant, $i$ is the imaginary unit, $A: \mathbb{R}^{2} \rightarrow \mathbb{R}^{2}$ is the magnetic potential.

When one looks for standing wave solutions $u(x, t):=e^{-i E t / \hbar} u(x)$, with $E \in \mathbb{R}$, of Eq. (1.8), the problem can be reduced by

$$
\left(\frac{\varepsilon}{i} \nabla-A(x)\right)^{2} u+V(x) u=f\left(|u|^{2}\right) u \text { in } \mathbb{R}^{N} .
$$


As far as we know, the first result seems to be established in [23], where the existence of standing waves to problem (1.9) has been obtained for $\hbar>0$ fixed and for special classes of magnetic fields. In this way, the authors obtained the existence of solutions by solving an appropriate minimization problem for the corresponding energy functional in the cases $N=2$ and $N=3$. After that, Kurata [31] proved that the problem has a least energy solution for any $\epsilon>0$ when a technical condition relating $V(x)$ and $A(x)$ is assumed. Under this technical condition, Kurata proved that the associated energy functional satisfies the Palais-Smale condition at any level. In [3], by combining a local assumption on $V$, the penalization techniques of del Pino and Felmer [19] and the Ljusternik-Schnirelmann theory, Alves et al. obtained the multiple solutions. We would like to refer to [16-18,22,28,35] for other results related with the problem (1.9).

Recently, there are many works concerning the following magnetic Schrödinger equation with deepening potential well

$$
-(\nabla+\mathrm{i} A(x))^{2} u+(\lambda V(x)+Z(x)) u=f\left(|u|^{2}\right) u \text { in } \mathbb{R}^{N} .
$$

In particular, Tang [39] considered multi-bump solutions of problem (1.10) with critical frequency in which $Z(x) \equiv 0$ and $f$ satisfies subcritical growth. Liang and Shi [33] considered multi-bump solutions of problem (1.10) with critical nonlinearity for the case $N \geq 3$. It is quite natural to consider multi-bump solutions for the problem when the nonlinearity satisfies the exponential critical growth in $N=2$. To the best of our knowledge, this problem has not been considered. Motivated by $[3,8,33]$, the main goal of the present paper is to prove the existence of multi-bump solutions for problem (1.10), considering a class of nonlinearity with exponential critical growth in $\mathbb{R}^{2}$. Because the nonlinearity has exponential critical growth in $\mathbb{R}^{2}$, some properties that are valid for $N \geq 3$, do not necessarily hold for the class of problems studied in this paper. Therefore, we need to take different approaches in some estimates. On the other hand, as we will see later, due to the presence of the magnetic field $A(x)$, problem (1.10) cannot be changed into a pure real-valued problem, hence we should deal with a complex-valued directly, which causes several new difficulties in employing the methods in dealing with our problem. Our problem is more complicated than the pattern studied in [8] and we need additional technical estimates.

We now present the general assumptions used in the statement of the main result of this paper.

(A) $A: \mathbb{R}^{2} \rightarrow \mathbb{R}^{2}$ is in $L_{\text {loc }}^{2}\left(\mathbb{R}^{2}\right)$.

$\left(V_{1}\right) V(x) \in C\left(\mathbb{R}^{2}, \mathbb{R}\right)$ with $V(x) \geq 0$.

$\left(V_{2}\right)$ The potential well $\Omega=$ int $V^{-1}(0)$ is a non-empty bounded open set with smooth boundary $\partial \Omega$ and $\bar{\Omega}=V^{-1}(0), \Omega$ can be decomposed in $k$ connected components $\Omega_{1}, \ldots, \Omega_{k}$ with $\operatorname{dist}\left(\Omega_{i}, \Omega_{j}\right)>0, i \neq j$.

$\left(V_{3}\right)$ There exist two positive constants $M_{0}$ and $M_{1}$ such that

$$
\lambda V(x)+Z(x) \geq M_{0}, \quad \forall x \in \mathbb{R}^{2}, \lambda \geq 1,
$$

and

$$
|Z(x)| \leq M_{1}, \quad \forall x \in \mathbb{R}^{2} .
$$


We assume that the reaction $f$ is a continuous function satisfying the following conditions.

$\left(f_{1}\right) f(t)=0$ if $t \leq 0$.

$\left(f_{2}\right)$ We have

$$
\lim _{t \rightarrow+\infty} \frac{f\left(t^{2}\right) t}{e^{\alpha t^{2}}}= \begin{cases}0, & \text { for } \alpha>4 \pi \\ +\infty, & \text { for } 0<\alpha<4 \pi .\end{cases}
$$

$\left(f_{3}\right)$ There is a positive constant $\theta>2$ such that

$$
0<\frac{\theta}{2} F(t) \leq t f(t), \quad \forall t>0,
$$

where $F(t)=\int_{0}^{t} f(s) d s$.

$\left(f_{4}\right)$ There exist constants $p>2$ and $C_{p}>0$ such that

$$
f(t) \geq C_{p} t^{(p-2) / 2} \text { for all } t>0,
$$

where

$$
\begin{aligned}
C_{p}>\left(\frac{k \theta(p-2)}{M^{*} p(\theta-2)}\right)^{(p-2) / 2} S_{p}^{p}, \quad M_{*}=\min \left\{1, M_{0}\right\}, \\
S_{p}=\max \left\{\inf _{\varphi \in H_{A}^{0,1}\left(\Omega_{j}\right) \backslash\{0\}} \frac{\left(\int_{\Omega_{j}}\left(\left|\nabla_{A} \varphi\right|^{2}+Z(x)|\varphi|^{2}\right) d x\right)^{1 / 2}}{\left(\int_{\Omega_{j}}|\varphi|^{p} d x\right)^{1 / p}}, j=1, \ldots, k\right\} .
\end{aligned}
$$

$\left(f_{5}\right) f(t)$ is an increasing function in $[0, \infty)$.

The main result in this paper is stated below.

Theorem 1.1. Assume that $(A),\left(V_{1}\right)-\left(V_{3}\right)$ and $\left(f_{1}\right)-\left(f_{5}\right)$ hold. Then, for any nonempty subset $\Gamma$ of $\{1,2, \ldots, k\}$, there exists $\lambda^{*}$ such that for all $\lambda \geq \lambda^{*}$, problem (1.10) has a nontrivial solution $u_{\lambda}$. Moreover, the family $\left\{u_{\lambda}\right\}_{\lambda \geq \lambda^{*}}$ has the following properties: for any sequence $\lambda_{n} \rightarrow \infty$, we can extract a subsequence $\lambda_{n_{i}}$ such that $u_{\lambda_{n_{i}}}$ converges strongly in $H_{A}^{1}\left(\mathbb{R}^{2}, \mathbb{C}\right)$ to a function $u$ which satisfies $u(x)=0$ for $x \notin \Omega_{\Gamma}$ and the restriction $\left.u\right|_{\Omega_{j}}$ is a least energy solution of

$$
\left\{\begin{array}{lc}
-(\nabla+\mathrm{i} A(x))^{2} u+Z(x) u=f\left(|u|^{2}\right) u, & \text { in } \Omega_{\Gamma}, \\
u=0, & \text { on } \partial \Omega_{j},
\end{array}\right.
$$

where $\Omega_{\Gamma}=\bigcup_{j \in \Gamma} \Omega_{j}$.

Corollary 1.2. Under the assumptions of Theorem 1.1, there exists $\lambda_{*}>0$ such that for all $\lambda \geq \lambda_{*}$, problem (1.10) has at least $2^{k}-1$ nontrivial solutions.

The paper is organized as follows. In Sect. 2 we introduce the functional setting and we give some preliminary results. In Sect. 3, we study the modified problem. We prove the Palais-Smale condition for the modified energy functional for $\lambda$ large and study $L^{\infty}$-estimates for the solution and the behavior of $(P S)_{\infty}$ sequences. In Sect. 4 , we adapt the deformation flow method in order to establish the existence of a special critical point, which is crucial for showing the existence of multi-bump solutions for $\lambda$ large enough and hence to prove Theorem 1.1. We refer to the recent monograph by Papageorgiou, Rădulescu and Repovš [38] for some of the abstract methods used in this paper. 


\section{Notation}

- $C, C_{1}, C_{2}, \ldots$ denote positive constants whose exact values are inessential and can change from line to line;

- $B_{R}(y)$ denotes the open disk centered at $y \in \mathbb{R}^{2}$ with radius $R>0$ and $B_{R}^{c}(y)$ denotes the complement of $B_{R}(y)$ in $\mathbb{R}^{2}$;

- $\|\cdot\|,\|\cdot\|_{q}$, and $\|\cdot\|_{L^{\infty}(\Omega)}$ denote the usual norms of the spaces $H^{1}\left(\mathbb{R}^{2}, \mathbb{R}\right)$, $L^{q}\left(\mathbb{R}^{2}, \mathbb{R}\right)$, and $L^{\infty}(\Omega, \mathbb{R})$, respectively, where $\Omega \subset \mathbb{R}^{2}$;

- $o_{n}(1)$ denotes a real sequence with $o_{n}(1) \rightarrow 0$ as $n \rightarrow+\infty$.

\section{Abstract setting and preliminary results}

In this section, we outline the variational framework for problem (1.10) and give some auxiliary properties.

For $u: \mathbb{R}^{2} \rightarrow \mathbb{C}$, let us denote by

$$
\nabla_{A} u:=(\nabla+i A) u .
$$

and

$$
H_{A}^{1}\left(\mathbb{R}^{2}, \mathbb{C}\right):=\left\{u \in L^{2}\left(\mathbb{R}^{2}, \mathbb{C}\right):\left|\nabla_{A} u\right| \in L^{2}\left(\mathbb{R}^{2}, \mathbb{R}\right)\right\} .
$$

The space $H_{A}^{1}\left(\mathbb{R}^{2}, \mathbb{C}\right)$ is a Hilbert space endowed with the scalar product

$$
\langle u, v\rangle:=\operatorname{Re} \int_{\mathbb{R}^{2}}\left(\nabla_{A} u \overline{\nabla_{A} v}+u \bar{v}\right) d x, \quad \text { for any } u, v \in H_{A}^{1}\left(\mathbb{R}^{2}, \mathbb{C}\right),
$$

where Re and the bar denote the real part of a complex number and the complex conjugation, respectively. Moreover, we denote by $\|u\|_{A}$ the norm induced by this inner product. The spaces $H_{A}^{1}\left(\mathbb{R}^{2}, \mathbb{C}\right)$ and $H^{1}\left(\mathbb{R}^{2}, \mathbb{R}\right)$ are not comparable, more precisely, in general $H_{A}^{1}\left(\mathbb{R}^{2}, \mathbb{C}\right) \not \subset H^{1}\left(\mathbb{R}^{2}, \mathbb{R}\right)$ and $H^{1}\left(\mathbb{R}^{2}, \mathbb{R}\right) \not \subset H_{A}^{1}\left(\mathbb{R}^{2}, \mathbb{C}\right)$.

By hypothesis $(A)$, on the space $H_{A}^{1}\left(\mathbb{R}^{2}, \mathbb{C}\right)$ we have the following diamagnetic inequality (see e.g. [32, Theorem 7.21]):

$$
\left|\nabla_{A} u(x)\right| \geq|\nabla| u(x)||
$$

Let

$$
E_{\lambda}\left(\mathbb{R}^{2}, \mathbb{C}\right):=\left\{u \in H_{A}^{1}\left(\mathbb{R}^{2}, \mathbb{C}\right): \int_{\mathbb{R}^{2}} \lambda V(x)|u|^{2} d x<\infty\right\},
$$

with the norm

$$
\|u\|_{\lambda}^{2}=\int_{\mathbb{R}^{2}}\left(\left|\nabla_{A} u\right|^{2}+(\lambda V(x)+Z(x))|u|^{2}\right) d x .
$$

For $\lambda \geq 1$, it is easy to see that $\left(E_{\lambda}\left(\mathbb{R}^{2}, \mathbb{C}\right),\|\cdot\|_{\lambda}\right)$ is a Hilbert space and $E_{\lambda}\left(\mathbb{R}^{2}, \mathbb{C}\right) \subset H_{A}^{1}\left(\mathbb{R}^{2}, \mathbb{C}\right)$.

Let $K \subset \mathbb{R}^{2}$ be an open set. We define 


$$
\begin{aligned}
H_{A}^{1}(K) & :=\left\{u \in L^{2}(K, \mathbb{C}):\left|\nabla_{A} u\right| \in L^{2}(K, \mathbb{R})\right\}, \\
\|u\|_{H_{A}^{1}(K)} & =\left(\int_{\mathbb{R}^{2}}\left(\left|\nabla_{A} u\right|^{2}+|u|^{2}\right) d x\right)^{\frac{1}{2}}, \\
E_{\lambda}(K, \mathbb{C}) & :=\left\{u \in H_{A}^{1}(K, \mathbb{C}): \int_{K} \lambda V(x)|u|^{2} d x<\infty\right\}, \\
\|u\|_{\lambda, K}^{2} & =\int_{K}\left(\left|\nabla_{A} u\right|^{2}+(\lambda V(x)+Z(x))|u|^{2}\right) d x .
\end{aligned}
$$

Let $H_{A}^{0,1}(K, \mathbb{C})$ be the Hilbert space defined by the closure of $C_{0}^{\infty}(K, \mathbb{C})$ under the norm $\|u\|_{H_{A}^{1}(K)}$.

The diamagnetic inequality (2.1) implies that if $u \in E_{\lambda}\left(\mathbb{R}^{2}, \mathbb{C}\right)$, then $|u| \in H^{1}\left(\mathbb{R}^{2}, \mathbb{R}\right)$ and $\|u\| \leq C\|u\|_{\lambda}$. Therefore, the embedding $E_{\lambda}\left(\mathbb{R}^{2}, \mathbb{C}\right) \hookrightarrow$ $L^{r}\left(\mathbb{R}^{2}, \mathbb{C}\right)$ is continuous for $r \geq 2$ and the embedding $E_{\lambda}\left(\mathbb{R}^{2}, \mathbb{C}\right) \hookrightarrow L_{\text {loc }}^{r}\left(\mathbb{R}^{2}, \mathbb{C}\right)$ is compact for $r \geq 1$.

Remark 2.1. Since the embedding $H_{A}^{1}\left(\mathbb{R}^{2}, \mathbb{C}\right) \hookrightarrow L_{\mathrm{loc}}^{r}\left(\mathbb{R}^{2}, \mathbb{C}\right)$ is compact for $r \geq$ 1 , a standard argument shows that the following infimum in $\left(f_{4}\right)$ is achieved and

$$
\inf _{\varphi \in H_{A}^{1}\left(\Omega_{j}\right) \backslash\{0\}} \frac{\left(\int_{\Omega_{j}}\left(\left|\nabla_{A} \varphi\right|^{2}+Z(x)|\varphi|^{2}\right) d x\right)^{1 / 2}}{\left(\int_{\Omega_{j}}|\varphi|^{p} d x\right)^{1 / p}}>0,
$$

for $j=1, \ldots, k$.

We recall that $u \in E_{\lambda}\left(\mathbb{R}^{2}, \mathbb{C}\right)$ is a weak solution of (1.10), if

$$
\operatorname{Re} \int_{\mathbb{R}^{2}}\left(\nabla_{A} u \overline{\nabla_{A} \phi}+(\lambda V(x)+Z(x)) u \bar{\phi}\right) d x=\operatorname{Re} \int_{\mathbb{R}^{2}} f\left(|u|^{2}\right) u \bar{\phi} d x,
$$

for all $\phi \in E_{\lambda}\left(\mathbb{R}^{2}, \mathbb{C}\right)$.

The weak solutions of problem (1.10) are the critical points of $I_{\lambda}$ : $E_{\lambda}\left(\mathbb{R}^{2}, \mathbb{C}\right) \rightarrow \mathbb{R}$ given by

$$
I_{\lambda}(u)=\frac{1}{2} \int_{\mathbb{R}^{2}}\left(\left|\nabla_{A} u\right|^{2}+(\lambda V(x)+Z(x))|u|^{2}\right) d x-\frac{1}{2} \int_{\mathbb{R}^{2}} F\left(|u|^{2}\right) d x,
$$

where $F(t)=\int_{0}^{t} f(s) d s$. It is easy to prove that $I_{\lambda} \in C^{1}\left(E_{\lambda}\left(\mathbb{R}^{2}, \mathbb{C}\right), \mathbb{R}\right)$.

In view of $\left(V_{3}\right)$, for any open set $K \subset \mathbb{R}^{2}$, it is easy to see that

$M_{0}\|u\|_{2, K}^{2} \leq \int_{K}\left(\left|\nabla_{A} u\right|^{2}+(\lambda V(x)+Z(x))|u|^{2}\right) d x$ for all $u \in E_{\lambda}(K)$, and $\lambda \geq 1$,

where $\|u\|_{2, K}^{2}=\int_{K}|u|^{2} d x$. The following property is an immediate consequence of the above consideration.

Lemma 2.2. There exist $\delta_{0}, v_{0}>0$ with $\delta_{0} \sim 1$ and $v_{0} \sim 0$ such that for any open set $K \subset \mathbb{R}^{N}$

$$
\delta_{0}\|u\|_{\lambda, K}^{2} \leq\|u\|_{\lambda, K}^{2}-v_{0}\|u\|_{2, K}^{2}, \text { for all } u \in E_{\lambda}(K, \mathbb{C}), \text { and } \lambda \geq 1 .
$$


The below estimates involving $f$ are the key points in this paper. By $\left(f_{1}\right)$ and $\left(f_{2}\right)$, fixed $q>2$, for any $\zeta>0$ and $\alpha>4 \pi$, there exists a constant $C>0$ depending on $q, \alpha, \zeta$, such that

$$
f(t) \leq \zeta+C t^{(q-2) / 2}\left(e^{\alpha t}-1\right) \text { for all } t \geq 0
$$

and, using $\left(f_{3}\right)$, we have

$$
F(t) \leq \zeta t+C t^{q / 2}\left(e^{\alpha t}-1\right) \text { for all } t \geq 0 .
$$

Moreover, by (2.2) and (2.3),

$$
f\left(t^{2}\right) t^{2} \leq \zeta t^{2}+C|t|^{q}\left(e^{\alpha t^{2}}-1\right) \text { for all } t \in \mathbb{R}
$$

and

$$
F\left(t^{2}\right) \leq \zeta t^{2}+C|t|^{q}\left(e^{\alpha t^{2}}-1\right) \text { for all } t \in \mathbb{R} .
$$

Now we recall a version of the Trudinger-Moser inequality in the whole space $\mathbb{R}^{2}$ due to Cao [15] (see also [13], Lemma 2.3) for functions belonging to $H^{1}\left(\mathbb{R}^{2}, \mathbb{R}\right)$.

Lemma 2.3. If $\alpha>0$ and $u \in H^{1}\left(\mathbb{R}^{2}, \mathbb{R}\right)$, then

$$
\int_{\mathbb{R}^{2}}\left(e^{\alpha u^{2}}-1\right) d x<+\infty .
$$

Moreover, if $\|\nabla u\|_{2}^{2} \leq 1,\|u\|_{2} \leq M<+\infty$, and $0<\alpha<4 \pi$, then there exists a positive constant $C(M, \alpha)$, which depends only on $M$ and $\alpha$, such that

$$
\int_{\mathbb{R}^{2}}\left(e^{\alpha u^{2}}-1\right) d x \leq C(M, \alpha) .
$$

To finish this section, in what follows, for each $j \in\{1,2, \ldots, k\}$, we fix a bounded open subset $\Omega_{j}^{\prime}$ with smooth boundary such that

(i) $\overline{\Omega_{j}} \subset \Omega_{j}^{\prime}$;

(ii) $\overline{\Omega_{i}^{\prime}} \cap \overline{\Omega_{j}^{\prime}}=\emptyset$ for all $i \neq j$.

\section{An auxiliary problem}

Since $\mathbb{R}^{2}$ is unbounded, we know that the Sobolev embeddings are not compact, as so $I_{\lambda}$ cannot verify the Palais-Smale condition. In order to overcome this difficulty, we adapt an argument of the penalization method introduced by del Pino and Felmer [19] and Ding and Tanaka [21].

Let $\nu_{0}>0$ be a constant given in Lemma 2.2, $\kappa>\frac{\theta}{\theta-2}>1$ and $a>0$ verifying $f(a)=\frac{\nu_{0}}{\kappa}$ and $\tilde{f}, \widetilde{F}: \mathbb{R} \rightarrow \mathbb{R}$ given by

$$
\tilde{f}(t)= \begin{cases}f(t), & t \leq a, \\ \frac{\nu_{0}}{\kappa}, & t \geq a,\end{cases}
$$


and

$$
\widetilde{F}(t)=\int_{0}^{t} \tilde{f}(s) d s
$$

Note that

$$
\widetilde{f}(t) \leq f(t), \quad t \geq 0 .
$$

From now on, we fix a non-empty subset $\Gamma \subset\{1, \ldots, k\}$ and

$$
\begin{gathered}
\Omega_{\Gamma}=\bigcup_{j \in \Gamma} \Omega_{j}, \quad \Omega_{\Gamma}^{\prime}=\bigcup_{j \in \Gamma} \Omega_{j}^{\prime}, \\
\chi_{\Gamma}(x):= \begin{cases}1 & \text { for } x \in \Omega_{\Gamma}^{\prime}, \\
0 & \text { for } x \notin \Omega_{\Gamma}^{\prime},\end{cases}
\end{gathered}
$$

the function

$$
\begin{aligned}
g(x, t) & =\chi_{\Gamma}(x) f(t)+\left(1-\chi_{\Gamma}(x)\right) \tilde{f}(t) \\
G(x, t) & =\int_{0}^{t} g(x, s) d s=\chi_{\Gamma}(x) F(t)+\left(1-\chi_{\Gamma}(x)\right) \tilde{F}(t) .
\end{aligned}
$$

It follows from (3.1) that $g$ satisfies the following inequality

$$
g\left(x,|u|^{2}\right)|u|^{2} \leq f\left(|u|^{2}\right)|u|^{2} .
$$

Let $\Phi_{\lambda}: E_{\lambda}\left(\mathbb{R}^{2}, \mathbb{C}\right) \rightarrow \mathbb{R}$ be the functional defined by

$$
\Phi_{\lambda}(u)=\frac{1}{2} \int_{\mathbb{R}^{2}}\left(\left|\nabla_{A} u\right|^{2}+(\lambda V(x)+Z(x))|u|^{2}\right) d x-\frac{1}{2} \int_{\mathbb{R}^{2}} G\left(x,|u|^{2}\right) d x .
$$

Standard arguments show that $\Phi_{\lambda} \in C^{1}\left(E_{\lambda}\left(\mathbb{R}^{2}, \mathbb{C}\right), \mathbb{R}\right)$ and its critical points are weak solutions of

$$
-(\nabla+\mathrm{i} A(x))^{2} u+(\lambda V(x)+Z(x)) u=g\left(x,|u|^{2}\right) u, \quad x \in \mathbb{R}^{2} .
$$

Our aim is to obtain nontrivial solutions of (3.5) which are solutions of the original problem (1.10). More precisely, if $u_{\lambda}$ is a nontrivial solution of (3.5) verifying $\left|u_{\lambda}(x)\right|^{2} \leq a$ in $\mathbb{R}^{2} \backslash \Omega_{\Gamma}^{\prime}$, then it is a nontrivial solution to (1.10).

\subsection{The Palais-Smale condition and consequences}

We start this subsection studying the boundedness of the Palais-Smale sequence related to $\Phi_{\lambda}$, that is, a sequence $\left(u_{n}\right) \subset E_{\lambda}\left(\mathbb{R}^{2}, \mathbb{C}\right)$ verifying

$$
\Phi_{\lambda}\left(u_{n}\right) \rightarrow c \text { and } \Phi_{\lambda}^{\prime}\left(u_{n}\right) \rightarrow 0
$$

for some $c \in \mathbb{R}$ (shortly $\left(u_{n}\right)$ is a $(P S)_{c}$ sequence). 
Lemma 3.1. If $\left(u_{n}\right)$ is a $(P S)_{c}$ sequence to $\Phi_{\lambda}$, it follows that

$$
\limsup _{n \rightarrow \infty}\left\|u_{n}\right\|_{\lambda}^{2} \leq\left(\frac{1}{2}-\frac{1}{\theta}\right)^{-1} \delta_{0}^{-1} c
$$

where $\delta_{0}$ is given in Lemma 2.2.

Proof. From the definition of Palais-Smale sequence, we have

$$
\Phi_{\lambda}\left(u_{n}\right)-\frac{1}{\theta} \Phi_{\lambda}^{\prime}\left(u_{n}\right) u_{n}=c+o_{n}(1)+o_{n}(1)\left\|u_{n}\right\|_{\lambda}
$$

On the other hand, from (3.6), (3.2), $\kappa>\theta /(\theta-2),\left(f_{3}\right)$ and Lemma 2.2, we obtain

$$
\begin{aligned}
\Phi_{\lambda}\left(u_{n}\right)-\frac{1}{\theta} \Phi_{\lambda}^{\prime}\left(u_{n}\right) u_{n}= & \left(\frac{1}{2}-\frac{1}{\theta}\right) \int_{\mathbb{R}^{2}}\left(\left|\nabla_{A} u_{n}\right|^{2}+(\lambda V(x)+Z(x))\left|u_{n}\right|^{2}\right) d x \\
& +\int_{\mathbb{R}^{2}}\left(\frac{1}{\theta} g\left(x,\left|u_{n}\right|^{2}\right)\left|u_{n}\right|^{2}-\frac{1}{2} G\left(x,\left|u_{n}\right|^{2}\right)\right) d x \\
\geq & \left(\frac{1}{2}-\frac{1}{\theta}\right) \int_{\mathbb{R}^{2}}\left(\left|\nabla_{A} u_{n}\right|^{2}+(\lambda V(x)+Z(x))\left|u_{n}\right|^{2}\right) d x \\
& +\int_{\mathbb{R}^{2} \backslash \Omega_{\Gamma}^{\prime}}\left(\frac{1}{\theta} \tilde{f}\left(\left|u_{n}\right|^{2}\right)\left|u_{n}\right|^{2}-\frac{1}{2} \tilde{F}\left(\left|u_{n}\right|^{2}\right)\right) d x \\
\geq & \left(\frac{1}{2}-\frac{1}{\theta}\right) \int_{\mathbb{R}^{2}}\left(\left|\nabla_{A} u_{n}\right|^{2}+(\lambda V(x)+Z(x))\left|u_{n}\right|^{2}\right) d x \\
& -\frac{1}{2} \int_{\mathbb{R}^{2} \backslash \Omega_{\Gamma}^{\prime}} \tilde{F}\left(\left|u_{n}\right|^{2}\right) d x \\
\geq & \left(\frac{1}{2}-\frac{1}{\theta}\right) \int_{\mathbb{R}^{2}}\left(\left|\nabla_{A} u_{n}\right|^{2}+(\lambda V(x)+Z(x))\left|u_{n}\right|^{2}\right) d x \\
& -\frac{v_{0}}{2 \kappa} \int_{\mathbb{R}^{2}}\left|u_{n}\right|^{2} d x \\
\geq & \left(\frac{1}{2}-\frac{1}{\theta}\right)\left(\left\|u_{n}\right\|_{\lambda}^{2}-v_{0}\left\|u_{n}\right\|_{2}^{2}\right) \\
\geq & \left(\frac{1}{2}-\frac{1}{\theta}\right) \delta_{0}\left\|u_{n}\right\|_{\lambda}^{2} .
\end{aligned}
$$

Therefore,

$$
\left(\frac{1}{2}-\frac{1}{\theta}\right) \delta_{0}\left\|u_{n}\right\|_{\lambda}^{2} \leq c+o_{n}(1)+o_{n}(1)\left\|u_{n}\right\|_{\lambda}
$$

This shows that $\left(u_{n}\right)$ is bounded and

$$
\limsup _{n \rightarrow \infty}\left\|u_{n}\right\|_{\lambda}^{2} \leq\left(\frac{1}{2}-\frac{1}{\theta}\right)^{-1} \delta_{0}^{-1} c,
$$

which completes the proof. 
For each fixed $j \in \Gamma$, let us denote by $c_{j}$ the minimax level of the functional $I_{j}: H_{A}^{0,1}\left(\Omega_{j}\right) \rightarrow \mathbb{R}$ given by

$$
I_{j}(u)=\frac{1}{2} \int_{\Omega_{j}}\left(\left|\nabla_{A} u\right|^{2}+Z(x)|u|^{2}\right) d x-\frac{1}{2} \int_{\Omega_{j}} F\left(|u|^{2}\right) d x .
$$

and

$$
c_{j}=\inf _{\gamma \in \Lambda_{j}} \max _{t \in[0,1]} I_{j}(\gamma(t))
$$

where

$$
\Lambda_{j}=\left\{\gamma \in C\left([0,1], H_{A}^{0,1}\left(\Omega_{j}, \mathbb{C}\right)\right): \gamma(0)=0, I_{j}(\gamma(1))<0\right\} .
$$

It is well known that the critical points of $I_{j}$ are weak solutions of the following problem

$$
\begin{cases}-(\nabla+\mathrm{i} A(x))^{2} u+Z(x) u=f\left(x,|u|^{2}\right) u, & \text { in } \Omega_{j}, \\ u=0, & \text { on } \partial \Omega_{j} .\end{cases}
$$

In the next lemma, we denote by $S$ the following real number

$$
S=\sum_{j=1}^{k} c_{j}
$$

Lemma 3.2. If $\left(f_{1}\right)-\left(f_{5}\right)$ hold, then $0<S<M^{*} \delta_{0}\left(\frac{1}{2}-\frac{1}{\theta}\right)$.

Proof. For each $j \in\{1, \ldots, k\}$, we may choose a function $\varphi_{j} \in H_{A}^{0,1}\left(\Omega_{j}, \mathbb{C}\right)$ such that

$$
\begin{aligned}
S_{p, j}= & \min _{\varphi \in H_{A}^{0,1}\left(\Omega_{j}\right) \backslash\{0\}} \frac{\left(\int_{\Omega_{j}}\left(\left|\nabla_{A} \varphi\right|^{2}+Z(x)|\varphi|^{2}\right) d x\right)^{1 / 2}}{\left(\int_{\Omega_{j}}|\varphi|^{p} d x\right)^{1 / p}} \\
= & \frac{\left(\int_{\Omega_{j}}\left(\left|\nabla_{A} \varphi_{j}\right|^{2}+Z(x)\left|\varphi_{j}\right|^{2}\right) d x\right)^{1 / 2}}{\left(\int_{\Omega_{j}}\left|\varphi_{j}\right|^{p} d x\right)^{1 / p}}
\end{aligned}
$$

Notice that

$$
\begin{aligned}
c_{j} & \leq \max _{t \geq 0} I_{j}\left(t \varphi_{j}\right) \\
& \leq \max _{t \geq 0}\left(\frac{t^{2}}{2} \int_{\Omega_{j}}\left(\left|\nabla_{A} \varphi_{j}\right|^{2}+Z(x)\left|\varphi_{j}\right|^{2}\right) d x-\frac{t^{p} C_{p}}{p} \int_{\Omega_{j}}\left|\varphi_{j}\right|^{p} d x\right) \\
& =\frac{p-2}{2 p} \frac{S_{p, j}^{\frac{2 p}{p-2}}}{C_{p}^{\frac{2}{p-2}}}
\end{aligned}
$$




$$
\leq \frac{p-2}{2 p} \frac{S_{p}^{\frac{2 p}{p-2}}}{C_{p}^{\frac{2}{p-2}}}
$$

Hence

$$
S=\sum_{j=1}^{k} c_{j} \leq k \frac{p-2}{2 p} \frac{S_{p}^{\frac{2 p}{p-2}}}{C_{p}^{\frac{2}{p-2}}} .
$$

On the other hand, by $\left(f_{4}\right)$ we have

$$
k \frac{p-2}{2 p} \frac{S_{p}^{\frac{2 p}{p-2}}}{C_{p}^{\frac{2}{p-2}}}<\left(\frac{1}{2}-\frac{1}{\theta}\right) M^{*} .
$$

Since $\delta_{0}$ may be chosen close to 1 , the last inequality implies that

$$
S<M^{*} \delta_{0}\left(\frac{1}{2}-\frac{1}{\theta}\right)
$$

This completes the proof of the lemma.

Proposition 3.3. For any $\lambda \geq 1$, the functional $\Phi_{\lambda}$ satisfies the $(P S)_{c}$ condition for all $c \in(0, S]$, that is, if $c \in(0, S]$, any $(P S)_{c}$-sequence $\left(u_{n}\right) \subset E_{\lambda}\left(\mathbb{R}^{2}, \mathbb{C}\right)$ of $\Phi_{\lambda}$ has a strongly convergent subsequence in $E_{\lambda}\left(\mathbb{R}^{2}, \mathbb{C}\right)$.

Proof. Let $\left(u_{n}\right) \subset E_{\lambda}\left(\mathbb{R}^{2}, \mathbb{C}\right)$ be a $(P S)_{c}$-sequence for $\Phi_{\lambda}$ at the level $c \in(0, S]$. By Lemmas 3.1 and 3.2 we obtain

$$
\limsup _{n \rightarrow \infty}\left\|u_{n}\right\|_{\lambda}^{2}<1
$$

Thus, up to a subsequence, $u_{n} \rightarrow u$ in $E_{\lambda}\left(\mathbb{R}^{2}, \mathbb{C}\right)$ and $u_{n} \rightarrow u$ in $L_{\text {loc }}^{q}\left(\mathbb{R}^{2}, \mathbb{C}\right)$ for all $q \geq 1$ as $n \rightarrow+\infty$. Moreover, by (3.6) and (2.2), fixed $q>2$, for any $\zeta>0$ and $\alpha>4 \pi$, there exists a constant $C>0$, which depends on $q, \alpha, \zeta$, such that for any $\phi \in E_{\lambda}\left(\mathbb{R}^{2}, \mathbb{C}\right)$,

$$
\left|\operatorname{Re} \int_{\mathbb{R}^{2}} g\left(x,\left|u_{n}\right|^{2}\right) u_{n} \bar{\phi} d x\right| \leq \zeta \int_{\mathbb{R}^{2}}\left|u_{n}\right||\bar{\phi}| d x+C \int_{\mathbb{R}^{2}}|\bar{\phi}|\left|u_{n}\right|^{q-1}\left(e^{\alpha\left|u_{n}\right|^{2}}-1\right) d x .
$$

Arguing as in [18, Lemma 2.5], we have

$$
\operatorname{Re} \int_{\mathbb{R}^{2}} g\left(x,\left|u_{n}\right|^{2}\right) u_{n} \bar{\phi} d x \rightarrow \operatorname{Re} \int_{\mathbb{R}^{2}} g\left(\varepsilon x,|u|^{2}\right) u \bar{\phi} d x .
$$

Thus, $u$ is a critical point of $\Phi_{\lambda}$.

Now, we take $R>0$ such that $\Omega_{\Gamma}^{\prime} \subset B_{\frac{R}{2}}(0)$. Let $\phi_{R} \in C^{\infty}\left(\mathbb{R}^{2}, \mathbb{R}\right)$ be a cut-off function such that

$\phi_{R}=0 \quad x \in B_{\frac{R}{2}}(0), \quad \phi_{R}=1 \quad x \in B_{R}^{c}(0), \quad 0 \leq \phi_{R} \leq 1, \quad$ and $\quad\left|\nabla \phi_{R}\right| \leq C / R$, 
where $C>0$ is a constant independent of $R$. By a direct computation, one has

$$
<\Phi_{\lambda}^{\prime}\left(u_{n}\right), u_{n} \phi_{R}>\rightarrow 0
$$

and

$$
\overline{\nabla_{A}\left(u_{n} \phi_{R}\right)}=\overline{u_{n}} \nabla \phi_{R}+\phi_{R} \overline{\nabla_{A} u_{n}} .
$$

Therefore,

$$
\begin{aligned}
o_{n}(1)=<\Phi_{\lambda}^{\prime}\left(u_{n}\right), u_{n} \phi_{R}>= & \int_{\mathbb{R}^{2}}\left(\left|\nabla_{A} u_{n}\right|^{2} \phi_{R}+(\lambda V(x)+Z(x))\left|u_{n}\right|^{2} \phi_{R}\right) d x \\
& +\operatorname{Re}\left(\int_{\mathbb{R}^{2}} \overline{u_{n}} \nabla_{A} u_{n} \nabla \phi_{R} d x\right) \\
& -\int_{\mathbb{R}^{2}} \tilde{f}\left(\left|u_{n}\right|^{2}\right)\left|u_{n}\right|^{2} \phi_{R} d x .
\end{aligned}
$$

Notice that

$$
\begin{aligned}
\left|\operatorname{Re}\left(\overline{u_{n}} \nabla_{A} u_{n}\right)\right| & =\left|\operatorname{Re}\left(\left(\nabla u_{n}+i A u_{n}\right) \overline{u_{n}}\right)\right|=\left|\operatorname{Re}\left(\overline{u_{n}} \nabla u_{n}\right)\right| \\
& =\left|u_{n}\right|\left|\operatorname{Re}\left(\frac{\overline{u_{n}}}{\left|u_{n}\right|} \nabla u_{n}\right)\right|=\left|u_{n}\right||\nabla| u_{n}|| .
\end{aligned}
$$

Using the Hölder inequality and (3.7) we obtain

$$
\limsup _{n \rightarrow \infty}\left|\operatorname{Re}\left(\int_{\mathbb{R}^{2}} \overline{u_{n}} \nabla_{A} u_{n} \nabla \phi_{R} d x\right)\right| \leq \frac{C}{R} .
$$

Moreover, we have

$$
\begin{aligned}
& \int_{\mathbb{R}^{2}}\left(\left|\nabla_{A} u_{n}\right|^{2} \phi_{R}+(\lambda V(x)+Z(x))\left|u_{n}\right|^{2} \phi_{R}\right) d x \\
& \leq \int_{\mathbb{R}^{2}} \tilde{f}\left(\left|u_{n}\right|^{2}\right)\left|u_{n}\right|^{2} \phi_{R} d x+\frac{C}{R}+o_{n}(1) \\
& \leq \frac{\nu_{0}}{\kappa} \int_{\mathbb{R}^{2}}\left|u_{n}\right|^{2} \phi_{R} d x+\frac{C}{R}+o_{n}(1),
\end{aligned}
$$

which implies that for any $\zeta>0$, there exists $R^{*}>0$ large, if $R>R^{*}$, one has

$$
\limsup _{n \rightarrow \infty} \int_{\mathbb{R}^{2} \backslash B_{R}(0)}\left(\left|\nabla_{A} u_{n}\right|^{2}+(\lambda V(x)+Z(x))\left|u_{n}\right|^{2}\right) d x \leq \zeta .
$$

Similarity, by (3.6) and (2.2), fixed $q>2$, for any $\zeta>0$ and $\alpha>4 \pi$, there exists a constant $C>0$, which depends on $q, \alpha, \zeta$, such that

$$
g\left(x,\left|u_{n}\right|^{2}\right)\left|u_{n}\right|^{2} \leq \zeta\left|u_{n}\right|^{2}+C\left|u_{n}\right|^{q}\left(e^{\alpha\left|u_{n}\right|^{2}}-1\right) .
$$

Since $u_{n} \rightarrow u$ in $L_{\mathrm{loc}}^{r}\left(\mathbb{R}^{2}, \mathbb{C}\right)$, for all $r \geq 1$, up to a subsequence, we have that

$$
\left|u_{n}\right| \rightarrow|u| \text { a.e. in } \mathbb{R}^{2} \text { as } n \rightarrow+\infty
$$


Then

$$
g\left(x,\left|u_{n}\right|^{2}\right)\left|u_{n}\right|^{2} \rightarrow g\left(x,|u|^{2}\right)|u|^{2} \text { a.e. in } \mathbb{R}^{2} \text { as } n \rightarrow+\infty \text {. }
$$

Moreover, $\left|u_{n}\right| \rightarrow|u|$ in $L_{\text {loc }}^{r}\left(\mathbb{R}^{2}, \mathbb{R}\right)$ for all $r \geq 1$.

Let

$$
P(x, t):=g\left(x, t^{2}\right) t \text { and } Q(t):=e^{\alpha t^{2}}-1, \quad t \in \mathbb{R},
$$

where $\alpha>4 \pi$ with $\alpha\left\|\left|u_{n}\right|\right\|<4 \pi$ for $n$ large. Using (3.6) and $\left(f_{2}\right)$, it is easy to see that

$$
\lim _{t \rightarrow+\infty} \frac{P(x, t)}{Q(t)}=0 \text { uniformly for } x \in \mathbb{R}^{2}
$$

and by Lemma 2.3,

$$
\sup _{n} \int_{\mathbb{R}^{2}} Q\left(\left|u_{n}\right|\right) d x \leq C .
$$

Then [14, Theorem A.I] implies

$$
\left.\lim _{n} \int_{B_{R}(0)}\left|g\left(x,\left|u_{n}\right|^{2}\right)\right| u_{n}\right|^{2}-g\left(x,|u|^{2}\right)|u|^{2} \mid d x=0 .
$$

Moreover, by (3.8) and the definition of $g$, we have

$$
\begin{aligned}
& \left.\int_{B_{R}^{c}(0)}\left|g\left(x,\left|u_{n}\right|^{2}\right)\right| u_{n}\right|^{2}-g\left(x,|u|^{2}\right)|u|^{2} \mid d x \\
& \quad \leq \frac{2 v_{0}}{\kappa} \int_{B_{R}^{c}(0)}\left(\left|\nabla_{A} u_{n}\right|^{2}+(\lambda V(x)+Z(x))\left|u_{n}\right|^{2}\right) d x<\frac{2 v_{0} \zeta}{\kappa}
\end{aligned}
$$

for any $\zeta>0$.

Hence

$$
\int_{\mathbb{R}^{2}} g\left(x,\left|u_{n}\right|^{2}\right)\left|u_{n}\right|^{2} d x \rightarrow \int_{\mathbb{R}^{2}} g\left(x,|u|^{2}\right)|u|^{2} d x \text { as } n \rightarrow+\infty .
$$

Finally, since $\Phi_{\lambda}^{\prime}(u)=0$, we have

$o_{n}(1)=\Phi_{\lambda}^{\prime}\left(u_{n}\right)\left[u_{n}\right]=\left\|u_{n}\right\|_{\lambda}^{2}-\int_{\mathbb{R}^{2}} g\left(x,\left|u_{n}\right|^{2}\right)\left|u_{n}\right|^{2} d x=\left\|u_{n}\right\|_{\lambda}^{2}-\|u\|_{\lambda}^{2}+o_{n}(1)$.

Thus, the sequence $\left(u_{n}\right)$ strong converges to $u$ in $E_{\lambda}\left(\mathbb{R}^{2}, \mathbb{C}\right)$.

Our next step is to study the behavior of a $(P S)_{\infty, c}$ sequence, that is, a sequence $\left(u_{n}\right) \subset H_{A}^{1}\left(\mathbb{R}^{2}, \mathbb{C}\right)$ satisfying

$$
\begin{aligned}
& u_{n} \in E_{\lambda_{n}}\left(\mathbb{R}^{2}, \mathbb{C}\right) \text { and } \lambda_{n} \rightarrow \infty, \\
& \Phi_{\lambda_{n}}\left(u_{n}\right) \rightarrow c, \\
& \left\|\Phi_{\lambda_{n}}^{\prime}\left(u_{n}\right)\right\|_{E_{\lambda_{n}}^{*}} \rightarrow 0, \text { as } n \rightarrow \infty .
\end{aligned}
$$


Proposition 3.4. Let $\left(u_{n}\right)$ be a $(P S)_{\infty, c}$ sequence with $c \in(0, S]$. Then, for some subsequence, still denoted by $\left(u_{n}\right)$, there exists $u \in H_{A}^{1}\left(\mathbb{R}^{2}, \mathbb{C}\right)$ such that

$$
u_{n} \rightarrow u \text { weakly in } H_{A}^{1}\left(\mathbb{R}^{2}, \mathbb{C}\right) \text {. }
$$

\section{Moreover,}

(i) $u \equiv 0$ in $\mathbb{R}^{2} \backslash \Omega_{\Gamma}$ and $\left.u\right|_{\Omega_{j}}$ is a solution of (3.6), for $\forall j \in \Gamma$;

(ii) $\left\|u_{n}-u\right\|_{\lambda_{n}} \rightarrow 0$;

(iii) $u_{n}$ also satisfies

$$
\begin{aligned}
& \lambda_{n} \int_{\mathbb{R}^{2}} V(x)\left|u_{n}\right|^{2} d x \rightarrow 0, \\
& \left\|u_{n}-u\right\|_{\lambda_{n}, \mathbb{R}^{2} \backslash \Omega_{\Gamma}}^{2} \rightarrow 0, \\
& \left\|u_{n}\right\|_{\lambda_{n}, \mathbb{R}^{2} \backslash \Omega_{j}^{\prime}}^{2} \rightarrow \int_{\Omega_{j}}\left(\left|\nabla_{A} u\right|^{2}+Z(x)|u|^{2}\right) d x .
\end{aligned}
$$

Proof. As in the proof of Proposition 3.3, it is easy to check that

$$
\limsup _{n \rightarrow \infty}\left\|u_{n}\right\|_{\lambda_{n}}^{2}<1 .
$$

Thus $\left(u_{n}\right)$ is bounded in $H_{A}^{1}\left(\mathbb{R}^{2}, \mathbb{C}\right)$ and we may assume that for some $u \in$ $H_{A}^{1}\left(\mathbb{R}^{2}, \mathbb{C}\right)$, up to a subsequence, if necessary

$$
\begin{aligned}
& u_{n} \rightarrow u \text { weakly in } H_{A}^{1}\left(\mathbb{R}^{2}, \mathbb{C}\right), \\
& u_{n} \rightarrow u \text { strongly in } L_{\text {loc }}^{r}\left(\mathbb{R}^{2}, \mathbb{C}\right), \quad \forall r \geq 1, \\
& \left|u_{n}\right| \rightarrow|u| \text { a.e. in } \mathbb{R}^{2} .
\end{aligned}
$$

To show (i), we fix the set $C_{m}=\left\{x \in \mathbb{R}^{2}: V(x) \geq \frac{1}{m}\right\}$. Then, for $n$ large

$$
\begin{aligned}
\int_{C_{m}}\left|u_{n}\right|^{2} d x & \leq \frac{m}{\lambda_{n}} \int_{\mathbb{R}^{2}} \lambda_{n} V(x)\left|u_{n}\right|^{2} d x \\
& \leq \frac{2 m}{\lambda_{n}} \int_{\mathbb{R}^{2}}\left(\left|\nabla_{A} u_{n}\right|^{2}+\left(\lambda_{n} V(x)+Z(x)\right)\left|u_{n}\right|^{2}\right) d x \\
& =\frac{2 m}{\lambda_{n}}\left\|u_{n}\right\|_{\lambda_{n}}^{2} .
\end{aligned}
$$

The last inequality together with Fatou's lemma imply

$$
\int_{C_{m}}|u|^{2} d x=0, \forall m \in \mathbb{N} .
$$

Therefore, $u(x)=0$ on $\bigcup_{m=1}^{+\infty} C_{m}=\mathbb{R}^{2} \backslash \Omega$, from which we can assert that $\left.u\right|_{\Omega_{j}} \in$ $H_{A}^{0,1}\left(\Omega_{j}, \mathbb{C}\right)$ for any $j \in\{1,2, \ldots, k\}$. 
Since $\Phi_{\lambda_{n}}^{\prime}\left(u_{n}\right) \varphi \rightarrow 0$ as $n \rightarrow \infty$, for each $\varphi \in C_{0}^{\infty}\left(\Omega_{j}, \mathbb{C}\right)$ (and hence for each $\left.\varphi \in H_{A}^{0,1}\left(\Omega_{j}, \mathbb{C}\right)\right)$, from (3.9) and the similar arguments in Proposition 3.3, we have

$$
\left.\operatorname{Re}\left(\int_{\Omega_{j}}\left(\mid \nabla_{A} u \overline{\nabla_{A} \varphi}+Z(x) u \bar{\varphi}\right) d x-\int_{\Omega_{j}} g\left(x,|u|^{2}\right) u \bar{\varphi}\right) d x\right)=0,
$$

showing that $\left.u\right|_{\Omega_{j}}$ is a solution of problem (3.6) for each $j \in\{1, \ldots, k\}$.

For each $j \in\{1, \ldots, k\} \backslash \Gamma$, setting $\varphi=\left.u\right|_{\Omega_{j}}$ in (3.11), we have

$$
\int_{\Omega_{j}}\left(\left|\nabla_{A} u\right|^{2}+Z(x)|u|^{2}\right) d x-\int_{\Omega_{j}} \tilde{f}\left(|u|^{2}\right)|u|^{2} d x=0 .
$$

By Lemma 2.2 and the fact that $\tilde{f}\left(t^{2}\right) t^{2} \leq \frac{\nu_{0}}{\kappa} t^{2}$ for all $t \in \mathbb{R}$, it yields

$$
\begin{aligned}
\delta_{0}\|u\|_{\lambda, \Omega_{j}}^{2} & \leq\|u\|_{\lambda, \Omega_{j}}^{2}-\frac{\nu_{0}}{k}\|u\|_{2, \Omega_{j}}^{2} \\
& \leq \int_{\Omega_{j}}\left(\left|\nabla_{A} u\right|^{2}+Z(x)|u|^{2}\right) d x-\int_{\Omega_{j}} \tilde{f}\left(|u|^{2}\right)|u|^{2} d x=0 .
\end{aligned}
$$

Thus $u=0$ in $\Omega_{j}$ for $j \in\{1,2, \ldots, k\} \backslash \Gamma$, it means that (i) holds.

For (ii), using the similar arguments in the proof of Proposition 3.3, for each $\zeta>0$, there exists $R>0$ such that

$$
\int_{\mathbb{R}^{2} \backslash B_{R}(0)}\left(\left|\nabla_{A} u_{n}\right|^{2}+\left(\lambda_{n} V(x)+Z(x)\right)\left|u_{n}\right|^{2}\right) d x \leq \zeta, \quad \text { for } n \in N .
$$

Using the same arguments as in the proof of Proposition 3.3 and (i), the above inequality implies that

$$
\begin{aligned}
& \int_{\mathbb{R}^{2}} g\left(x,\left|u_{n}\right|^{2}\right)\left|u_{n}\right|^{2} d x \rightarrow \int_{\mathbb{R}^{2}} g\left(x,|u|^{2}\right)|u|^{2} d x \\
& =\int_{\Omega_{\Gamma}} f\left(|u|^{2}\right)|u|^{2} d x \text { as } n \rightarrow+\infty,
\end{aligned}
$$

Now, by (i) again, we have

$$
\begin{aligned}
o_{n}(1) & =\Phi_{\lambda_{n}}^{\prime}\left(u_{n}\right)\left[u_{n}\right]=\left\|u_{n}\right\|_{\lambda}^{2}-\int_{\mathbb{R}^{2}} g\left(x,\left|u_{n}\right|^{2}\right)\left|u_{n}\right|^{2} d x \\
& =\left\|u_{n}\right\|_{\lambda_{n}}^{2}-\|u\|_{\lambda_{n}}^{2}+o_{n}(1) .
\end{aligned}
$$

Thus, the sequence $\left(u_{n}\right)$ strong converges to $u$ in $E_{\lambda_{n}}\left(\mathbb{R}^{2}, \mathbb{C}\right)$ and (ii) holds.

To prove (iii), notice that from (i) and (ii),

$$
\begin{aligned}
\lambda_{n} \int_{\mathbb{R}^{2}} V(x)\left|u_{n}\right|^{2} d x & =\lambda_{n} \int_{\mathbb{R}^{2}} V(x)\left|u_{n}-u\right|^{2} d x \\
& \leq C\left\|u_{n}-u\right\|_{\lambda_{n}}^{2} \rightarrow 0 \text { as } n \rightarrow \infty .
\end{aligned}
$$


Moreover, from (i) and (ii), it is also easy to obtain that as $n \rightarrow \infty$

$$
\begin{aligned}
& \left\|u_{n}-u\right\|_{\lambda_{n}, \mathbb{R}^{2} \backslash \Omega_{\Gamma}}^{2} \rightarrow 0, \\
& \left\|u_{n}\right\|_{\lambda_{n}, \mathbb{R}^{2} \backslash \Omega_{j}^{\prime}}^{2} \rightarrow \int_{\Omega_{j}}\left(\left|\nabla_{A} u\right|^{2}+Z(x)|u|^{2}\right) d x \text { for all } j \in \Gamma .
\end{aligned}
$$

Therefore, the proof is complete.

Proposition 3.5. For each $\lambda \geq 1$, let $u_{\lambda}$ be a nontrivial solution of problem (3.5) with $\left\|u_{\lambda}\right\|_{\lambda}^{2}<1$. Then, there exists $K, \lambda^{*}>0$ such that

$$
\left\|\left|u_{\lambda}\right|\right\|_{L^{\infty}\left(\mathbb{R}^{2}\right)}^{2} \leq K \quad \forall \lambda \geq \lambda^{*}
$$

Proof. Let $\left(\lambda_{n}\right)$ be a sequence with $\lambda_{n} \rightarrow \infty$ and define $u_{n}(x)=u_{\lambda_{n}}(x)$. For any $R>0$ and $0<r \leq R / 2$, let $\eta \in C^{\infty}\left(\mathbb{R}^{2}\right), 0 \leq \eta \leq 1$ with $\eta(x)=1$ if $|x| \geq R$ and $\eta(x)=0$ if $|x| \leq R-r$ and $|\nabla \eta| \leq 2 / r$.

For each $n \in N$ and $L>0$, we consider the functions

$$
u_{L, n}(x):=\left\{\begin{array}{ll}
\left|u_{n}(x)\right| & \text { if }\left|u_{n}(x)\right| \leq L, \\
L & \text { if }\left|u_{n}(x)\right|>L,
\end{array} z_{L, n}:=\eta^{2} u_{L, n}^{2(\beta-1)} u_{n}, \quad \text { and } \quad w_{L, n}:=\eta u_{L, n}^{\beta-1}\left|u_{n}\right|,\right.
$$

where $\beta>1$ will be determined later.

By straightforward computations, we have

$$
\overline{\nabla_{A} z_{L, n}}=\eta^{2} u_{L, n}^{2(\beta-1)} \overline{\nabla_{A} u_{n}}+\overline{u_{n}} \nabla\left(\eta^{2} u_{L, n}^{2(\beta-1)}\right)
$$

and

$$
\nabla_{A} u_{n} \overline{\nabla_{A} z_{L, n}}=\left|\nabla_{A} u_{n}\right|^{2} \eta^{2} u_{L, n}^{2(\beta-1)}+\overline{u_{n}} \nabla_{A} u_{n} \nabla\left(\eta^{2} u_{L, n}^{2(\beta-1)}\right)
$$

Taking the real part of $\nabla_{A} u_{n} \overline{\nabla_{A} z_{L, n}}$ and using the diamagnetic inequality (2.1), we obtain

$$
\begin{aligned}
\operatorname{Re}\left(\nabla_{A} u_{n} \overline{\nabla_{A} z_{L, n}}\right) & =\left|\nabla_{A} u_{n}\right|^{2} \eta^{2} u_{L, n}^{2(\beta-1)}+\operatorname{Re}\left(\overline{u_{n}} \nabla_{A} u_{n} \nabla\left(\eta^{2} u_{L, n}^{2(\beta-1)}\right)\right) \\
& \geq|\nabla| u_{n}||^{2} \eta^{2} u_{L, n}^{2(\beta-1)}+\left|u_{n}\right| \nabla\left|u_{n}\right| \nabla\left(\eta^{2} u_{L, n}^{2(\beta-1)}\right) \\
& \geq\left.|\nabla| u_{n}\right|^{2} \eta^{2} u_{L, n}^{2(\beta-1)}+2 \eta \nabla \eta u_{L, n}^{2(\beta-1)}\left|u_{n}\right| \nabla\left|u_{n}\right| .
\end{aligned}
$$

Taking $z_{L, n}$ as the test function, we have

$\operatorname{Re} \int_{\mathbb{R}^{2}}\left(\nabla_{A} u_{n} \overline{\nabla_{A} z_{L, n}}+(\lambda V(x)+Z(x)) u_{\lambda} \overline{z_{L, n}}\right) d x=\operatorname{Re} \int_{\mathbb{R}^{2}} g\left(x,\left|u_{n}\right|^{2}\right) u_{n} \overline{z_{L, n}} d x$. 
By (3.12), the Young inequality (with $\tau>0$ ), (3.6), (2.4), for $\alpha>4 \pi$ and for a fixed $q>2$, given $0<\zeta<M_{0}$, there exists $C>0$ such that

$$
\begin{aligned}
& \int_{\mathbb{R}^{2}}|\nabla| u_{n}||^{2} \eta^{2} u_{L, n}^{2(\beta-1)} d x \leq \int_{\mathbb{R}^{2}}|\nabla| u_{n}||^{2} \eta^{2} u_{L, n}^{2(\beta-1)} d x \\
& +2 \int_{\mathbb{R}^{2}} \eta \nabla \eta u_{L, n}^{2(\beta-1)}\left|u_{n}\right| \nabla\left|u_{n}\right| d x \\
& +\int_{\mathbb{R}^{2}}(\lambda V(x)+Z(x)) u_{L, n}^{2(\beta-1)} \eta^{2}\left|u_{n}\right|^{2} d x \\
& +2 \int_{\mathbb{R}^{2}} \eta|\nabla \eta| u_{L, n}^{2(\beta-1)}\left|u_{n}\right||\nabla| u_{n}|| \\
& -\zeta \int_{\mathbb{R}^{2}} u_{L, n}^{2(\beta-1)} \eta^{2}\left|u_{n}\right|^{2} d x \\
& \leq \operatorname{Re} \int_{\mathbb{R}^{2}}\left(\nabla_{A} u_{n} \overline{\nabla_{A} z_{L, n}}+(\lambda V(x)+Z(x)) u_{n} \overline{z_{L, n}}\right) d x \\
& +\tau \int_{\mathbb{R}^{2}}|\nabla| u_{n}||^{2} \eta^{2} u_{L, n}^{2(\beta-1)} d x \\
& +\frac{1}{\tau} \int_{\mathbb{R}^{2}}|\nabla \eta|^{2} u_{L, n}^{2(\beta-1)}\left|u_{n}\right|^{2} d x \\
& -\zeta \int_{\mathbb{R}^{2}} u_{L, n}^{2(\beta-1)} \eta^{2}\left|u_{n}\right|^{2} d x \\
& \leq \int_{\mathbb{R}^{2}} g\left(x,\left|u_{n}\right|^{2}\right) \eta^{2}\left|u_{n}\right|^{2} u_{L, n}^{2(\beta-1)} d x \\
& +\tau \int_{\mathbb{R}^{2}}|\nabla| u_{n}||^{2} \eta^{2} u_{L, n}^{2(\beta-1)} d x \\
& +\frac{4}{\tau r^{2}} \int_{R-r \leq|x| \leq R} u_{L, n}^{2(\beta-1)}\left|u_{n}\right|^{2} d x \\
& -\zeta \int_{\mathbb{R}^{2}} \eta^{2} u_{L, n}^{2(\beta-1)}\left|u_{n}\right|^{2} d x \\
& \leq C \int_{\mathbb{R}^{2}}\left|u_{n}\right|^{q}\left(e^{\alpha\left|u_{n}\right|^{2}}-1\right) \eta^{2} u_{L, n}^{2(\beta-1)} d x \\
& +\tau \int_{\mathbb{R}^{2}}|\nabla| u_{n}||^{2} \eta^{2} u_{L, n}^{2(\beta-1)} d x \\
& +\frac{4}{\tau r^{2}} \int_{R-r \leq|x| \leq R}\left|u_{n}\right|^{2 \beta} d x \text {. }
\end{aligned}
$$

Hence, choosing $\tau>0$ sufficiently small, we get

$$
\begin{aligned}
\int_{\mathbb{R}^{2}}|\nabla| u_{n}||^{2} \eta^{2} u_{L, n}^{2(\beta-1)} \leq & C\left[\int_{|x| \geq R-r}\left|u_{n}\right|^{q+2(\beta-1)}\left(e^{\alpha\left|u_{n}\right|^{2}}-1\right) d x\right. \\
& \left.+\frac{1}{r^{2}} \int_{R-r \leq|x| \leq R}\left|u_{n}\right|^{2 \beta} d x\right] .
\end{aligned}
$$


Moreover, arguing similarly to (3.13), we can conclude that

$$
\begin{aligned}
\int_{\mathbb{R}^{2}} \eta^{2} u_{L, n}^{2(\beta-1)}\left|u_{n}\right|^{2} d x \leq C & {\left[\int_{|x| \geq R-r}\left|u_{n}\right|^{q+2(\beta-1)}\left(e^{\alpha\left|u_{n}\right|^{2}}-1\right) d x\right.} \\
& \left.+\frac{1}{r^{2}} \int_{R-r \leq|x| \leq R}\left|u_{n}\right|^{2 \beta} d x\right] .
\end{aligned}
$$

On the other hand, using the Sobolev embedding, (3.14), (3.15), the Hölder inequality with $t, \sigma, \tau>1,1 / \sigma+1 / \tau=1 / t, \sigma(q-2) \geq 2$, and the inequality $\left(e^{t}-1\right)^{s} \leq e^{t s}-1$, for $s>1$ and $t \geq 0$, we have

$$
\begin{aligned}
\left\|w_{L, n}\right\|_{q}^{2} \leq & C \int_{\mathbb{R}^{2}}\left(\left|\nabla w_{L, n}\right|^{2}+\left|w_{L, n}\right|^{2}\right) d x \\
\leq & C\left(\int_{\mathbb{R}^{2}}|\nabla \eta|^{2}\left|u_{n}\right|^{2 \beta} d x+\beta^{2} \int_{\mathbb{R}^{2}} \eta^{2} u_{L, n}^{2(\beta-1)}|\nabla| u_{n}||^{2} d x\right. \\
& \left.+\int_{\mathbb{R}^{2}} \eta^{2} u_{L, n}^{2(\beta-1)}\left|u_{n}\right|^{2} d x\right) \\
\leq & C \beta^{2}\left(\frac{1}{r^{2}} \int_{R-r \leq|x| \leq R}\left|u_{n}\right|^{2 \beta} d x\right. \\
& +\int_{|x| \geq R-r}\left|u_{n}\right|^{q+2(\beta-1)}\left(e^{\left.\left.\alpha\left|u_{n}\right|^{2}-1\right) d x\right)}\right. \\
\leq & C \beta^{2}\left[\frac{R^{2 / t}}{r^{2}}+\left(\int_{|x| \geq R-r}\left|u_{n}\right|^{\sigma(q-2)} d x\right)^{1 / \sigma}\right. \\
& \left.\left(\int_{\mathbb{R}^{2}}\left(e^{\tau \alpha\left|u_{n}\right|^{2}}-1\right) d x\right)^{1 / \tau}\right] \\
& \left(\int_{|x| \geq R-r}\left|u_{n}\right|^{2 \beta t /(t-1)} d x\right)^{(t-1) / t} .
\end{aligned}
$$

Since $\left(u_{n}\right) \subset H_{A}^{1}\left(\mathbb{R}^{2}, \mathbb{C}\right)$ is a a $(P S)_{\infty, c}$ sequence, up to a subsequence, by Proposition 3.4, we have $u_{n} \rightarrow u$ in $H_{A}^{1}\left(\mathbb{R}^{2}, \mathbb{C}\right)$. By (3.16), it follows that

$\left(\int_{|x| \geq R} u_{L, n}^{q \beta} d x\right)^{2 / q} \leq\left\|w_{L, n}\right\|_{q}^{2} \leq C \beta^{2}\left(1+\frac{R^{2 / t}}{r^{2}}\right)\left(\int_{|x| \geq R-r}\left|u_{n}\right|^{2 \beta t /(t-1)}\right)^{(t-1) / t}$

and, applying the Fatou's Lemma as $L \rightarrow+\infty$, we obtain

$$
\left(\int_{|x| \geq R}\left|u_{n}\right|^{q \beta} d x\right)^{2 / q} \leq C \beta^{2}\left(1+\frac{R^{2 / t}}{r^{2}}\right)\left(\int_{|x| \geq R-r}\left|u_{n}\right|^{2 \beta t /(t-1)}\right)^{(t-1) / t} .
$$

Next, if we take $\zeta:=\frac{q(t-1)}{2 t}, \beta:=\zeta^{m}$, with $m \in \mathbb{N}^{*}$, and $s:=\frac{2 t}{t-1}$, we obtain

$$
\begin{aligned}
& \left(\int_{|x| \geq R}\left|u_{n}\right|^{s \zeta^{m+1}} d x\right)^{1 /\left(s \zeta^{m+1}\right)} \\
& \quad \leq C^{\zeta^{-m}} \zeta^{m \zeta^{-m}}\left(1+\frac{R^{2 / t}}{r^{2}}\right)^{1 /\left(2 \zeta^{m}\right)}\left(\int_{|x| \geq R-r}\left|u_{n}\right|^{s \zeta^{m}}\right)^{1 /\left(s \zeta^{m}\right)}
\end{aligned}
$$


for every $m \in \mathbb{N}^{*}$. Then, for $r=r_{m}:=R / 2^{m}, m \in \mathbb{N}^{*}$, using also that $2 / t<2$, we get

$$
\begin{aligned}
& \left(\int_{|x| \geq R}\left|u_{n}\right|^{s \zeta^{m+1}} d x\right)^{1 /\left(s \zeta^{m+1}\right)} \\
& \leq\left(\int_{|x| \geq R-r_{m+1}}\left|u_{n}\right|^{s \zeta^{m+1}} d x\right)^{1 /\left(s \zeta^{m+1}\right)} \\
& \leq C^{\sum_{i=1}^{m} \zeta^{-i}} \zeta^{\sum_{i=1}^{m} i \zeta^{-i}} \exp \left(\sum_{i=1}^{m} \frac{\ln \left(1+2^{2(i+1)}\right)}{2 \zeta^{i}}\right)\left(\int_{|x| \geq R / 2}\left|u_{n}\right|^{s \zeta} d x\right)^{1 /(s \zeta)} .
\end{aligned}
$$

Hence, passing to the limit as $m \rightarrow+\infty$ in the last inequality, we obtain

$$
\left\|u_{n}\right\|_{L^{\infty}\left(B_{R}^{c}(0)\right)} \leq C\left(\int_{|x| \geq R}\left|u_{n}\right|^{q} d x\right)^{1 / q} .
$$

For $x_{0} \in \mathbb{R}^{2}$, we can use the same argument taking $\eta \in C_{0}^{\infty}\left(\mathbb{R}^{2},[0,1]\right)$ with $\eta(x)=1$ if $\left|x-x_{0}\right| \leq \tilde{\rho}, \eta(x)=0$ if $\left|x-x_{0}\right|>2 \rho$, with $\tilde{\rho}<\rho$, and $|\nabla \eta| \leq 2 / \tilde{\rho}$, to prove that

$$
\left\|u_{n}\right\|_{L^{\infty}\left(\overline{B_{2 \rho}\left(x_{0}\right)}\right)} \leq C\left(\int_{|x| \leq 2 \rho}\left|u_{n}\right|^{q} d x\right)^{1 / q} .
$$

Thus, by (3.17), (3.18), and using a standard covering argument and the boundedness of $\left(\left|u_{\lambda}\right|\right)$ in $L^{q}\left(\mathbb{R}^{2}, \mathbb{R}\right)$, it follows that there exists $K>0$ such that

$$
\left\|\left|u_{n}\right|\right\|_{L^{\infty}\left(\mathbb{R}^{2}\right)} \leq K \quad \forall n \text { large. }
$$

Hence the proof is complete.

Proposition 3.6. Let $\left(u_{\lambda}\right)$ be a family of nontrivial solutions of problem (3.3) with $\left\|u_{\lambda}\right\|_{\lambda}^{2}<1$ and $\lambda \geq 1$. Then, there exists $\lambda^{*}>0$ such that

$$
\left\|\left|u_{\lambda}\right|\right\|_{L^{\infty}\left(\mathbb{R}^{2} \backslash \Omega_{\Gamma}^{\prime}\right)}^{2} \leq a, \quad \forall \lambda \geq \lambda^{*} .
$$

Proof. We use notation $B_{r}(x)=\left\{y \in \mathbb{R}^{2}:|x-y|<r\right\}$. Since $u_{\lambda} \in E_{\lambda}\left(\mathbb{R}^{2}, \mathbb{C}\right)$ is a critical point of $\Phi_{\lambda}(u)$, that is, $u_{\lambda}$ satisfies the following equation

$$
-(\nabla+\mathrm{i} A(x))^{2} u_{\lambda}+(\lambda V(x)+Z(x)) u_{\lambda}=g\left(x,\left|u_{\lambda}\right|^{2}\right) u_{\lambda}, \quad x \in \mathbb{R}^{2} .
$$

By Kato's inequality

$$
\Delta\left|u_{\lambda}\right| \geq \operatorname{Re}\left(\frac{\overline{u_{\lambda}}}{\left|u_{\lambda}\right|}(\nabla+i A(x))^{2} u_{\lambda}(x)\right),
$$

there holds

$$
\Delta\left|u_{\lambda}(x)\right|-(\lambda V(x)+Z(x))\left|u_{\lambda}(x)\right|-g\left(x,\left|u_{\lambda}\right|^{2}\right)\left|u_{\lambda}(x)\right| \geq 0, \quad x \in \mathbb{R}^{2},
$$

since $\left|u_{\lambda}\right| \geq 0$ and $(\lambda V(x)+Z(x)) \geq M_{0}>0$ if $\lambda \geq 1$, we have

$$
\Delta\left|u_{\lambda}(x)\right|-g\left(x,\left|u_{\lambda}\right|^{2}\right)\left|u_{\lambda}(x)\right| \geq 0, \quad x \in \mathbb{R}^{2} .
$$


Using Proposition 3.5 and the subsolution estimate (see [26] Theorem 8.17), there exists a constant $C(r)$ such that

$$
\sup _{y \in B_{r}(x)}\left|u_{\lambda}(y)\right| \leq C(r)\left(\int_{B_{2 r}(x)}\left|u_{\lambda}\right|^{2} d y\right)^{1 / 2} .
$$

By Proposition 3.4, for any sequence $\lambda_{n} \rightarrow \infty$, we can extract a subsequence $\lambda_{n_{i}}$ such that

$$
u_{\lambda_{n_{i}}} \rightarrow u_{0} \in H_{A}^{0,1}\left(\Omega_{\Gamma}, \mathbb{C}\right) \text { strongly in } H_{A}^{1}\left(\mathbb{R}^{2}, \mathbb{C}\right) .
$$

In particular,

$$
u_{\lambda_{n_{i}}} \rightarrow 0 \text { strongly in } L^{2}\left(\mathbb{R}^{2} \backslash \overline{\Omega_{\Gamma}}, \mathbb{C}\right) .
$$

Since $\lambda_{n} \rightarrow \infty$ is arbitrary, we have

$$
u_{\lambda} \rightarrow 0 \text { strongly in } L^{2}\left(\mathbb{R}^{2} \backslash \overline{\Omega_{\Gamma}}, \mathbb{C}\right) \text { as } \lambda \rightarrow \infty .
$$

Thus, choosing $r \in\left(0, \operatorname{dist}\left(\Omega_{\Gamma}, \mathbb{R}^{2} \backslash \Omega_{\Gamma}^{\prime}\right)\right)$, we have uniformly in $x \in \mathbb{R}^{2} \backslash \Omega_{\Gamma}^{\prime}$ that

$$
\begin{aligned}
\left|u_{\lambda}(y)\right| & \leq C(r)\left\|u_{\lambda}\right\|_{L^{2}\left(B_{2 r}(x)\right)} \\
& \leq C(r) \mid B_{r}(x)\left\|u_{\lambda}\right\|_{L^{2}\left(\mathbb{R}^{2} \backslash \Omega_{\Gamma}^{\prime}\right)} \\
& \rightarrow 0 .
\end{aligned}
$$

The proof is now complete.

\section{The existence of multi-bump positive solutions}

In this section, for each $j \in \Gamma$, we denote by $\Phi_{\lambda, j}: H_{A}^{1}\left(\Omega_{j}^{\prime}, \mathbb{C}\right) \rightarrow \mathbb{R}$ the functional given by

$$
\Phi_{\lambda, j}(u)=\frac{1}{2} \int_{\Omega_{j}^{\prime}}\left(\left|\nabla_{A} u\right|^{2}+(\lambda V(x)+Z(x))|u|^{2}\right) d x-\frac{1}{2} \int_{\Omega_{j}^{\prime}} F\left(|u|^{2}\right) d x .
$$

It is easy to check that the functional $\Phi_{\lambda, j}$ satisfies the mountain pass geometry. In what follows, we denote by $c_{\lambda, j}$ the minimax level related to the above functional defined by

$$
c_{\lambda, j}=\inf _{\gamma \in \Lambda_{\lambda, j}} \max _{t \in[0,1]} \Phi_{\lambda, j}(\gamma(t)),
$$

where

$$
\Lambda_{\lambda, j}=\left\{\gamma \in C\left([0,1], H_{A}^{1}\left(\Omega_{j}^{\prime}, \mathbb{C}\right)\right): \gamma(0)=0, \Phi_{\lambda, j}(\gamma(1))<0\right\} .
$$

Therefore, there exist $\left(u_{n}\right) \subset H_{A}^{0,1}\left(\Omega_{j}, \mathbb{C}\right)$ and $\left(u_{\lambda, n}\right) \subset H_{A}^{1}\left(\Omega_{j}^{\prime}, \mathbb{C}\right)$ verifying

$$
I_{j}\left(u_{n}\right) \rightarrow c_{j} \text { and } I_{j}^{\prime}\left(u_{n}\right) \rightarrow 0
$$


and

$$
\Phi_{\lambda, j}\left(u_{\lambda, n}\right) \rightarrow c_{\lambda, j} \quad \text { and } \quad \Phi_{\lambda, j}^{\prime}\left(u_{\lambda, n}\right) \rightarrow 0 .
$$

From $\left(f_{1}\right)$ and $\left(f_{3}\right)-\left(f_{5}\right)$, it is easy to prove that

$$
\sup _{n \in N}\left\|u_{n}\right\|_{H_{A}^{0,1}\left(\Omega_{j}\right)}<1 \text { and } \sup _{n \in N}\left\|u_{\lambda, n}\right\|_{H_{A}^{1}\left(\Omega_{j}^{\prime}\right)}<1 \text {, }
$$

and these inequalities imply that $I_{j}$ and $\Phi_{\lambda, j}$ satisfy the $(P S)_{c_{j}}$ and $(P S)_{c_{\lambda, j}}$ conditions, respectively. Therefore, it is easy to prove that there exist two nontrivial functions $w_{j} \in H_{A}^{0,1}\left(\Omega_{j}\right)$ and $w_{\lambda, j} \in H_{A}^{1}\left(\Omega_{j}^{\prime}\right)$ verifying

$$
I_{j}\left(w_{\lambda, j}\right)=c_{j} \quad \text { and } \quad I_{j}^{\prime}\left(w_{\lambda, j}\right)=0
$$

and

$$
\Phi_{\lambda, j}\left(w_{\lambda, j}\right)=c_{\lambda, j} \quad \text { and } \quad \Phi_{\lambda, j}^{\prime}\left(w_{\lambda, j}\right)=0 .
$$

Moreover, we have the following lemma.

Lemma 4.1. The following assertions hold:

(i) $0<c_{\lambda, j} \leq c_{j}$ for $\lambda \geq 1$ and $j \in \Gamma$.

(ii) $c_{j}\left(c_{\lambda, j}\right.$ respectively) is a least energy level for $I_{j}(u)\left(\Phi_{\lambda, j}(u)\right.$ respectively), that is

$$
c_{j}=\inf \left\{I_{j}(u): u \in H_{A}^{0,1}\left(\Omega_{j}\right) \backslash\{0\}, I_{j}^{\prime}(u) u=0\right\},
$$

and

$$
c_{\lambda, j}=\inf \left\{\Phi_{\lambda, j}(u): u \in H_{A}^{1}\left(\Omega_{j}^{\prime}\right) \backslash\{0\}, \Phi_{\lambda, j}^{\prime}(u) u=0\right\} .
$$

(iii) $c_{j}=\max _{t>0} I_{j}\left(t w_{j}\right), c_{\lambda, j}=\max _{t>0} \Phi_{\lambda, j}\left(t w_{\lambda, j}\right)$.

(iv) $c_{\lambda, j} \rightarrow c_{j}$ as $\lambda \rightarrow \infty$ for any $j \in \Gamma$.

Proof. From $\left(f_{4}\right)$, it is easy to prove that $c_{\lambda, j}>0$ and $c_{j}>0$ for any $j \in \Gamma$ and $\lambda \geq 1$.

Now for any $u \in H_{A}^{0,1}\left(\Omega_{j}\right)$, we may extend $u$ to $\tilde{u} \in H_{A}^{1}\left(\Omega_{j}^{\prime}\right)$ by

$$
\tilde{u}(x):= \begin{cases}u(x), & \text { in } \Omega_{j}, \\ 0 & \text { in } \Omega_{j}^{\prime} \backslash \overline{\Omega_{j}},\end{cases}
$$

and $H_{A}^{0,1}\left(\Omega_{j}\right) \subset H_{A}^{1}\left(\Omega_{j}^{\prime}\right)$. Thus, we have $\Lambda_{j} \subset \Lambda_{\lambda, j}$ and

$$
\begin{aligned}
c_{\lambda, j} & =\inf _{\gamma \in \Lambda_{\lambda, j}} \max _{t \in[0,1]} \Phi_{\lambda, j}(\gamma(t)) \\
& \leq \inf _{\gamma \in \Lambda_{j}} \max _{t \in[0,1]} \Phi_{\lambda, j}(\gamma(t)) \\
& =\inf _{\gamma \in \Lambda_{j}} \max _{t \in[0,1]} I_{j}(\gamma(t))=c_{j} .
\end{aligned}
$$

Thus (i) holds. The proof of (ii) and (iii) is standard by using the monotonicity of the term $f(t)$ with respect to $t$ for $t>0$. 
Now we prove (iv). Using Proposition 3.4, we may extract a subsequence $\lambda_{n} \rightarrow \infty$ such that

$$
w_{\lambda_{n}, j} \rightarrow u_{0} \text { strongly in } H_{A}^{1}\left(\Omega_{j}^{\prime}\right),
$$

where $u_{0} \in H_{A}^{0,1}\left(\Omega_{j}\right)$ is a solution of (3.6) and

$$
\Phi_{\lambda_{n}, j}\left(w_{\lambda_{n}, j}\right) \rightarrow I_{j}\left(u_{0}\right) .
$$

By the definition of $c_{j}$, we have

$$
\limsup _{\lambda \rightarrow \infty} c_{\lambda, j}=\limsup _{\lambda \rightarrow \infty} \Phi_{\lambda, j}\left(w_{\lambda, j}\right) \geq I_{j}\left(u_{0}\right) \geq c_{j} .
$$

Together with (i), we get (iv).

\subsection{A special critical value of $\Phi_{\lambda}$}

In what follows, let us fix $R>1$ such that

$$
\left|I_{j}\left(\frac{1}{R} w_{j}\right)\right|<\frac{1}{2} c_{j}, \forall j \in \Gamma
$$

and

$$
\left|I_{j}\left(R w_{j}\right)-c_{j}\right| \geq 1, \forall j \in \Gamma .
$$

From the definition of $c_{j}$, it is easy to check that

$$
\max _{s \in\left[1 / R^{2}, 1\right]} I_{j}\left(s R w_{j}\right)=c_{j}, \forall j \in \Gamma .
$$

We consider $\Gamma=\{1,2, \ldots, l\}(l \leq k)$, and the maps

$$
\begin{aligned}
& \gamma_{0}\left(s_{1}, s_{2}, \ldots, s_{l}\right)(x)=\sum_{j=1}^{l} s_{j} R w_{j}(x) \forall\left(s_{1}, s_{2}, \ldots, s_{l}\right) \in\left[1 / R^{2}, 1\right]^{l}, \\
& \Lambda_{*}=\left\{\gamma \in C\left(\left[1 / R^{2}, 1\right]^{l}, E_{\lambda}\left(\mathbb{R}^{2}, \mathbb{C}\right) \backslash\{0\}\right): \gamma=\gamma_{0} \text { on } \partial\left(\left[1 / R^{2}, 1\right]^{l}\right)\right\},
\end{aligned}
$$

and

$$
b_{\lambda, \Lambda}=\inf _{\gamma \in \Lambda_{*}} \max _{\left(s_{1}, s_{2}, \ldots, s_{l}\right) \in\left[1 / R^{2}, 1\right]^{l}} \Phi_{\lambda}\left(\gamma\left(s_{1}, s_{2}, \ldots, s_{l}\right)\right) .
$$

We remark that $\gamma_{0} \in \Lambda_{*}$, so $\Lambda_{*} \neq \emptyset$ and $b_{\lambda, \Lambda}$ is well defined.

Lemma 4.2. For any $\gamma \in \Lambda_{*}$, there exists $\left(t_{1}, t_{2}, \ldots, t_{l}\right) \in\left[1 / R^{2}, 1\right]^{l}$ such that $\Phi_{\lambda, j}^{\prime}\left(\gamma\left(t_{1}, t_{2}, \ldots, t_{l}\right)\right)\left(\gamma\left(t_{1}, t_{2}, \ldots, t_{l}\right)\right)=0$ for all $j \in\{1,2, \ldots, l\}$. 
Proof. For a given $\gamma \in \Lambda_{*}$, let us consider the map $\tilde{\gamma}:\left[1 / R^{2}, 1\right]^{l} \rightarrow \mathbb{R}^{l}$ defined by

$$
\tilde{\gamma}\left(s_{1}, s_{2}, \ldots, s_{l}\right)=\left(\Phi_{\lambda, 1}^{\prime}(\gamma)(\gamma), \Phi_{\lambda, 2}^{\prime}(\gamma)(\gamma), \ldots, \Phi_{\lambda, l}^{\prime}(\gamma)(\gamma)\right),
$$

where

$$
\Phi_{\lambda, j}^{\prime}(\gamma)(\gamma)=\Phi_{\lambda, j}^{\prime}\left(\gamma\left(s_{1}, s_{2}, \ldots, s_{l}\right)\right)\left(\gamma\left(s_{1}, s_{2}, \ldots, s_{l}\right)\right) \text { for all } j \in \Gamma .
$$

For any $\left(s_{1}, s_{2}, \ldots, s_{l}\right) \in \partial\left(\left[1 / R^{2}, 1\right]^{l}\right)$, it follows that

$$
\gamma\left(s_{1}, s_{2}, \ldots, s_{l}\right)=\gamma_{0}\left(s_{1}, s_{2}, \ldots, s_{l}\right) £ \neg
$$

and

$$
\Phi_{\lambda, j}^{\prime}\left(\gamma_{0}\left(s_{1}, s_{2}, \ldots, s_{l}\right)\right)\left(\gamma_{0}\left(s_{1}, s_{2}, \ldots, s_{l}\right)\right)=0 \Rightarrow s_{j} \notin\left\{1 / R^{2}, 1\right\}, \quad \forall j \in \Gamma .
$$

Thus,

$$
(0,0, \ldots, 0) \notin \tilde{\gamma}\left(\partial\left(\left[1 / R^{2}, 1\right]^{l}\right)\right)
$$

Using this fact, it follows from the topological degree

$$
\operatorname{deg}\left(\tilde{\gamma},\left(1 / R^{2}, 1\right)^{l},(0,0, \ldots, 0)\right)=(-1)^{l} \neq 0 .
$$

Hence, there exists $\left(t_{1}, t_{2}, \ldots, t_{l}\right) \in\left(1 / R^{2}, 1\right)^{l}$ satisfying

$$
\Phi_{\lambda, j}^{\prime}\left(\gamma\left(t_{1}, t_{2}, \ldots, t_{l}\right)\right)\left(\gamma\left(t_{1}, t_{2}, \ldots, t_{l}\right)\right)=0 \text { for all } j \in\{1,2, \ldots, l\} .
$$

The proof is thus completed.

In the sequel, let us denote by $c_{\Gamma}=\sum_{j=1}^{l} c_{j}$. From $\left(f_{4}\right)$, we know that

$$
c_{\Gamma} \in(0, S] \text {. }
$$

Proposition 4.3. The following facts hold

(i) $\sum_{j=1}^{l} c_{\lambda, j} \leq b_{\lambda, \Gamma} \leq c_{\Gamma}$ for all $\lambda \geq 1$.

(ii) $\Phi_{\lambda}\left(\gamma\left(s_{1}, s_{2}, \ldots, s_{l}\right)\right)<c_{\Gamma}$ for all $\lambda \geq 1, \gamma \in \Lambda_{*}$ and $\left(s_{1}, s_{2}, \ldots, s_{l}\right) \in$ $\partial\left(\left[1 / R^{2}, 1\right]^{l}\right)$.

Proof. Since $\gamma_{0}$ defined in (4.3) belongs to $\Lambda_{*}$, we have

$$
\begin{aligned}
b_{\lambda, \Gamma} & \leq \max _{\left(s_{1}, s_{2}, \ldots, s_{l}\right) \in\left[1 / R^{2}, 1\right]^{l}} \Phi_{\lambda}\left(\gamma_{0}\left(s_{1}, s_{2}, \ldots, s_{l}\right)\right) \\
& =\max _{\left(s_{1}, s_{2}, \ldots, s_{l}\right) \in\left[1 / R^{2}, 1\right]^{l}} \sum_{j=1}^{l} I_{j}\left(s R w_{j}\right) \\
& =\sum_{j=1}^{l} c_{j}=c_{\Gamma} .
\end{aligned}
$$


Fixing $\left(t_{1}, t_{2}, \ldots, t_{l}\right) \in\left[1 / R^{2}, 1\right]^{l}$ given in Lemma 4.2 and recalling that $c_{\lambda, j}$ can be characterized by

$$
c_{\lambda, j}=\inf \left\{\Phi_{\lambda, j}(u): u \in H_{A}^{1}\left(\Omega_{j}^{\prime}\right) \backslash\{0\}, \Phi_{\lambda, j}^{\prime}(u) u=0\right\} .
$$

It follows that

$$
\Phi_{\lambda, j}\left(\gamma\left(t_{1}, t_{2}, \ldots, t_{l}\right)\right) \geq c_{\lambda, j} \quad \forall j \in \Gamma .
$$

On the other hand, recalling that $\Phi_{\lambda, \mathbb{R}^{2} \backslash \Omega_{\Gamma}^{\prime}}(u) \geq 0$ for all $u \in H_{A}^{1}\left(\mathbb{R}^{2} \backslash \Omega_{\Gamma}^{\prime}\right)$, we have

$$
\Phi_{\lambda}\left(\gamma\left(s_{1}, s_{2}, \ldots, s_{l}\right)\right) \geq \sum_{j=1}^{l} \Phi_{\lambda, j}\left(\gamma\left(s_{1}, s_{2}, \ldots, s_{l}\right)\right) .
$$

Thus

$$
\max _{\left(s_{1}, s_{2}, \ldots, s_{l}\right) \in\left[1 / R^{2}, 1\right]^{l}} \Phi_{\lambda}\left(\gamma\left(s_{1}, s_{2}, \ldots, s_{l}\right)\right) \geq \Phi_{\lambda}\left(\gamma\left(t_{1}, t_{2}, \ldots, t_{l}\right)\right) \geq \sum_{j=1}^{l} c_{\lambda, j} .
$$

From the definition of $b_{\lambda, \Gamma}$, we can obtain

$$
b_{\lambda, \Gamma} \geq \sum_{j=1}^{l} c_{\lambda, j}
$$

This completes the proof of (i).

Since $\gamma\left(s_{1}, s_{2}, \ldots, s_{l}\right)=\gamma_{0}\left(s_{1}, s_{2}, \ldots, s_{l}\right)$ on $\partial\left(\left[1 / R^{2}, 1\right]^{l}\right)$, we have

$$
\Phi_{\lambda}\left(\gamma_{0}\left(s_{1}, s_{2}, \ldots, s_{l}\right)\right)=\sum_{j=1}^{l} I_{j}\left(s_{j} R w_{j}\right) .
$$

Moreover, $I_{j}\left(s_{j} R w_{j}\right) \leq c_{j}$ for all $j \in \Gamma$ and for some $j_{0} \in \Gamma, s_{j_{0}} \in\left\{1 / R^{2}, 1\right\}$ and $I_{j_{0}}\left(s_{j_{0}} R w_{j_{0}}\right) \leq \frac{c_{j_{0}}}{2}$. Therefore,

$$
\Phi_{\lambda}\left(\gamma_{0}\left(s_{1}, s_{2}, \ldots, s_{l}\right)\right) \leq c_{\Gamma}-\epsilon
$$

for some $\epsilon>0$. This completes the proof of (ii).

Corollary 4.4. The following claims hold:

(i) $b_{\lambda, \Gamma} \rightarrow c_{\Gamma}$ as $\lambda \rightarrow \infty$.

(ii) $b_{\lambda, \Gamma}$ is a critical value of $\Phi_{\lambda}$ for large $\lambda$.

Proof. (i) For all $\lambda \geq 1$ and for each $j$, we have $0<c_{\lambda, j} \leq c_{j}$. Using the same arguments in the proof of Proposition 3.4, we can prove that $c_{\lambda, j} \rightarrow c_{j}$ as $\lambda \rightarrow \infty$ and thus, from Proposition $4.3, b_{\lambda, \Gamma} \rightarrow c_{\Gamma}$ as $\lambda \rightarrow \infty$.

(ii) Using the fact that $\Phi_{\lambda}$ verifies that Palais-Smale condition, we can use well known arguments involving deformation lemma [40] to conclude that $b_{\lambda, \Gamma}$ is a critical level to $\Phi_{\lambda}$ for large $\lambda$. 


\subsection{Proof of the main result}

To prove Theorem 1.1, we need to find a nontrivial solution $u_{\lambda}$ for the large $\lambda$ which approaches a least energy solution in each $\Omega_{j}(j \in \Gamma)$ and to 0 elsewhere as $\lambda \rightarrow \infty$. To this end, we will show two propositions which imply together with the estimates made in the previous section that Theorem 1.1 holds.

Let

$$
\begin{aligned}
& M=1+\sum_{j=1}^{k} \sqrt{\left(\frac{1}{2}-\frac{1}{\theta}\right)^{-1} c_{j}}, \\
& \bar{B}_{M+1}(0)=\left\{u \in E_{\lambda}\left(\mathbb{R}^{2}, \mathbb{C}\right):\|u\|_{\lambda} \leq M+1\right\} .
\end{aligned}
$$

For small $\mu>0$, we define

$$
A_{\mu}^{\lambda}=\left\{u \in \bar{B}_{M+1}(0):\|u\|_{\lambda, \mathbb{R}^{2} \backslash \Omega_{j}^{\prime}} \leq \mu,\left|\Phi_{\lambda, j}(u)-c_{j}\right| \leq \mu, \forall j \in \Gamma\right\} .
$$

W also use the notation:

$$
\Phi_{\lambda}^{c_{\Gamma}}=\left\{u \in E_{\lambda}\left(\mathbb{R}^{2}, \mathbb{C}\right): \Phi_{\lambda}(u) \leq c_{\Gamma}\right\}
$$

and remark that $w=\sum_{j=1}^{l} w_{j} \in A_{\mu}^{\lambda} \cap \Phi_{\lambda}^{c_{\Gamma}}$, this shows that $A_{\mu}^{\lambda} \cap \Phi_{\lambda}^{c_{\Gamma}} \neq \emptyset$. Fixing

$$
0<\mu<\frac{1}{3} \min \left\{c_{j}, j \in \Gamma\right\} .
$$

We have the following uniform estimate of $\left\|\Phi_{\lambda}^{\prime}(u)\right\|_{\lambda}$ on the annulus $\left(A_{2 \mu}^{\lambda} \backslash A_{\mu}^{\lambda}\right) \cap$ $\Phi_{\lambda}^{c_{\Gamma}}$.

Proposition 4.5. Let $\mu>0$ satisfies (4.3). Then there exist $\sigma_{0}>0$ and $\lambda^{*} \geq 1$ independent of $\lambda$ such that

$$
\left\|\Phi_{\lambda}^{\prime}(u)\right\|_{\lambda} \geq \sigma_{0} \text { for } \lambda \geq \lambda^{*} \text { for all } u \in\left(A_{2 \mu}^{\lambda} \backslash A_{\mu}^{\lambda}\right) \cap \Phi_{\lambda_{n}}^{c_{\Gamma}} .
$$

Proof. Arguing by contradiction, we assume that there exist $\lambda_{n} \rightarrow \infty$ and $u_{n} \in$ $\left(A_{2 \mu}^{\lambda_{n}} \backslash A_{\mu}^{\lambda_{n}}\right) \cap \Phi_{\lambda_{n}}^{c_{\Gamma}}$ such that $\left\|\Phi_{\lambda}^{\prime}\left(u_{n}\right)\right\|_{\lambda_{n}} \rightarrow 0$.

Since $u_{n} \in A_{2 \mu}^{\lambda_{n}}$ and $\left\{\left\|u_{n}\right\|_{\lambda_{n}}\right\}$ is a bounded sequence, this shows that $\left\{\Phi_{\lambda_{n}}\left(u_{n}\right)\right\}$ is also bounded. Thus, we may assume that

$$
\Phi_{\lambda_{n}}\left(u_{n}\right) \rightarrow c \in\left(-\infty, c_{\Gamma}\right]
$$

up to a subsequence.

Applying Proposition 3.4, we can extract a subsequence $u_{n} \rightarrow u$ in $H_{A}^{1}\left(\mathbb{R}^{2}\right)$ where $u \in H_{A}^{0,1}\left(\Omega_{\Gamma}\right)$ is a solution of (4.1) with

$$
\begin{aligned}
& \lim _{n \rightarrow \infty} \Phi_{\lambda_{n}}\left(u_{n}\right)=\sum_{j=1}^{l} I_{j}(u) \leq c_{\Gamma}, \\
& \left\|u_{n}\right\|_{\lambda_{n}, \Omega_{j}^{\prime}}^{2} \rightarrow \int_{\Omega_{j}}\left(\left|\nabla_{A} u\right|^{2}+Z(x)|u|^{2}\right) d x, \text { for all } j \in \Gamma,
\end{aligned}
$$




$$
\begin{aligned}
& \lambda_{n} \int_{\mathbb{R}^{2}} V(x)\left|u_{n}\right|^{2} d x \rightarrow 0, \\
& \left\|u_{n}\right\|_{\lambda_{n}, \mathbb{R}^{2} \backslash \Omega_{\Gamma}}^{2} \rightarrow 0 .
\end{aligned}
$$

Since $c_{j}$ is the least energy level for $I_{j}$, we have two possibilities:

(i) $I_{j}\left(\left.u\right|_{\Omega_{j}}\right)=c_{j}$ for all $j \in \Gamma$.

(ii) $I_{j_{0}}\left(\left.u\right|_{\Omega_{j_{0}}}\right)=0$, that is $\left.u\right|_{\Omega_{j_{0}}} \equiv 0$ for some $j_{0} \in \Gamma$.

If (i) occurs, we have

$$
\frac{1}{2} \int_{\Omega_{j}}\left(\left|\nabla_{A} u\right|^{2}+Z(x)|u|^{2}\right) d x-\frac{1}{2} \int_{\Omega_{j}} F\left(|u|^{2}\right) d x=c_{j}, \quad \text { for all } j \in \Gamma .
$$

From (4.6)-(4.8), we have $u_{n} \in A_{\mu}^{\lambda_{n}}$ for large $n$, which is a contradiction to the assumption $u_{n} \in\left(A_{2 \mu}^{\lambda_{n}} \backslash A_{\mu}^{\lambda_{n}}\right)$.

If (ii) occurs, from (4.7) and $u_{n} \rightarrow u$ in $H_{A}^{1}\left(\mathbb{R}^{2}\right)$, it follows that

$$
\left|\Phi_{\lambda_{n}, j_{0}}\left(u_{n}\right)-c_{j_{0}}\right| \rightarrow c_{j_{0}} \geq 3 \mu
$$

which is a contradiction with the fact that $u_{n} \in\left(A_{2 \mu}^{\lambda_{n}} \backslash A_{\mu}^{\lambda_{n}}\right)$. Thus neither (i) nor (ii) can hold, and the proof is completed.

Proposition 4.6. Let $\mu>0$ satisfies (4.3) and $\lambda^{*} \geq 1$ be a constant given by in Proposition 4.5. Then, for $\lambda \geq \lambda^{*}$, there exists a nontrivial solution $u_{\lambda}$ of (3.5) satisfying $u_{\lambda} \in A_{\mu}^{\lambda} \cap \Phi_{\lambda}^{c_{\Gamma}}$.

Proof. Assuming by contradiction that there are no critical points in $A_{\mu}^{\lambda} \cap \Phi_{\lambda}^{c_{\Gamma}}$, since the Palais-Smale condition holds for $\Phi_{\lambda}$ in the energy level $(0, S]$, there exists a constant $d_{\lambda}>0$ such that

$$
\left\|\Phi_{\lambda}^{\prime}(u)\right\| \geq d_{\lambda} \text { for all } u \in A_{\mu}^{\lambda} \cap \Phi_{\lambda}^{c_{\Gamma}} .
$$

From hypothesis and Proposition 4.5, we also have

$$
\left\|\Phi_{\lambda}^{\prime}(u)\right\| \geq \sigma_{0} \text { for all } u \in\left(A_{2 \mu}^{\lambda} \backslash A_{\mu}^{\lambda}\right) \cap \Phi_{\lambda}^{c_{\Gamma}},
$$

where $\sigma_{0}>0$ is independent of $\lambda$. In what follows, $\Psi: E_{\lambda}\left(\mathbb{R}^{2}, \mathbb{C}\right) \rightarrow \mathbb{R}$ be a continuous functional that verify

$$
\begin{aligned}
\Psi(u)=1 \quad \text { for } \quad u \in A_{3 \mu / 2}^{\lambda}, \\
\Psi(u)=0 \quad \text { for } \quad u \notin A_{2 \mu}^{\lambda}, \\
0 \leq \Psi(u) \leq 1 \quad \text { for } \quad u \in E_{\lambda}\left(\mathbb{R}^{2}, \mathbb{C}\right),
\end{aligned}
$$

and $H: \Phi_{\lambda}^{c_{\Gamma}} \rightarrow \mathbb{R}$ be a continuous functional verify

$$
H(u):= \begin{cases}-\Psi(u) \frac{\Phi_{\lambda}^{\prime}(u)}{\left\|\Phi_{\lambda}^{\prime}(u)\right\|_{\lambda}} & u \in A_{2 \mu}^{\lambda}, \\ 0, & u \notin A_{2 \mu}^{\lambda} .\end{cases}
$$


Thus, we have the inequality

$$
\|H(u)\| \leq 1 \quad \forall \lambda \geq \Lambda_{*} \text { and } u \in \Phi_{\lambda}^{c_{\Gamma}} .
$$

Considering the deformation flow $\eta:[0, \infty) \times \Phi_{\lambda}^{c_{\Gamma}} \rightarrow \Phi_{\lambda}^{c_{\Gamma}}$ defined by

$$
\frac{d \eta}{d t}=H(\eta) \text { and } \eta(0, u)=u \in \Phi_{\lambda}^{c_{\Gamma}}
$$

Thus $\eta$ has the following properties

$$
\begin{aligned}
\frac{d}{d t} \Phi_{\lambda}(\eta(t, u)) & =-\Psi(\eta(t, u))\left\|\Phi_{\lambda}^{\prime}(\eta(t, u))\right\|_{\lambda}, \\
\eta(t, u) & =u \text { for all } t \geq 0 \text { and } u \in \Phi_{\lambda}^{C_{\Gamma}} \backslash A_{2 \mu}^{\lambda}, \\
\left|\Phi_{\lambda, j}(u)-\Phi_{\lambda, j}(v)\right| & \leq K^{*}\|u-v\|_{\lambda, \Omega_{j}^{\prime}} \quad \forall u, v \in \bar{B}_{M+1}(0) \text { and } j \in \Gamma,
\end{aligned}
$$

where $K^{*}>0$ be a constant.

Now let $\gamma_{0}\left(s_{1}, s_{2}, \ldots, s_{l}\right) \in \Lambda_{*}$ be a path defined in (4.4) and we consider $\eta\left(t, \gamma_{0}\left(s_{1}, s_{2}, \ldots, s_{l}\right)\right)$ for large $t$. Since for all $\left.\left(s_{1}, s_{2}, \ldots, s_{l}\right) \in \partial\left(\left[1 / R^{2}, 1\right]\right]^{l}\right)$, $\gamma_{0}\left(s_{1}, s_{2}, \ldots, s_{l}\right) \notin A_{2 \mu}^{\lambda}$, thus we have by (4.10) that

$\eta\left(t, \gamma_{0}\left(s_{1}, s_{2}, \ldots, s_{l}\right)\right)=\gamma_{0}\left(s_{1}, s_{2}, \ldots, s_{l}\right)$ for all $\left(s_{1}, s_{2}, \ldots, s_{l}\right) \in \partial\left(\left[1 / R^{2}, 1\right]^{l}\right)$ and $\eta\left(t, \gamma_{0}\left(s_{1}, s_{2}, \ldots, s_{l}\right)\right) \in \Lambda_{*}$ for all $t \geq 0$.

Since $\operatorname{supp} \gamma_{0}\left(s_{1}, s_{2}, \ldots, s_{l}\right)(x) \subset \bar{\Omega}_{\Gamma}$ for all $\left(s_{1}, s_{2}, \ldots, s_{l}\right) \in \partial\left(\left[1 / R^{2}, 1\right]\right)$, then $\Phi_{\lambda}\left(\gamma_{0}\left(s_{1}, s_{2}, \ldots, s_{l}\right)\right)$ and $\left\|\gamma_{0}\left(s_{1}, s_{2}, \ldots, s_{l}\right)\right\|_{\lambda, j}$ etc. do not depend on $\lambda \geq 0$. On the other hand,

$$
\Phi_{\lambda}\left(\gamma_{0}\left(s_{1}, s_{2}, \ldots, s_{l}\right)\right) \leq c_{\Gamma} \quad \forall\left(s_{1}, s_{2}, \ldots, s_{l}\right) \in\left[1 / R^{2}, 1\right]^{l}
$$

and $\Phi_{\lambda}\left(\gamma_{0}\left(s_{1}, s_{2}, \ldots, s_{l}\right)\right)=c_{\Gamma}$ if and only if $s_{j}=\frac{1}{R}$, that is $\gamma_{0}\left(s_{1}, s_{2}, \ldots, s_{l}\right)(x)$ $\left.\right|_{\Omega_{j}}=w_{j}$ for $j \in \Gamma$. Thus, we have that

$$
m_{0}:=\max \left\{\Phi_{\lambda}(u): u \in \gamma_{0}\left(\left[1 / R^{2}, 1\right]^{l}\right) \backslash A_{\mu}^{\lambda}\right\}
$$

is independent of $\lambda$ and $m_{0}<c_{\Gamma}$. Since $\left\|\frac{d \eta}{d t}\right\|_{\lambda} \leq 1$ for all $t, u$, it is easy to see that for any $t>0$

$$
\left\|\eta\left(0, \gamma_{0}\left(s_{1}, s_{2}, \ldots, s_{l}\right)\right)-\eta\left(t, \gamma_{0}\left(s_{1}, s_{2}, \ldots, s_{l}\right)\right)\right\|_{\lambda} \leq t
$$

Since $\Phi_{\lambda, j}(u) \in C^{1}\left(E_{\lambda}\left(\mathbb{R}^{2}, \mathbb{C}\right), \mathbb{R}\right)$ for all $j=1,2, \ldots, l$, and from the assumptions $\left(f_{1}\right)-\left(f_{5}\right)$, it is easy to see that for a large number $T>0$, there exists a positive number $\rho_{0}>0$ which is independent of $\lambda$ such that for all $j=1,2, \ldots, l$ and $t \in[0, T]$,

$$
\| \Phi_{\lambda, j}\left(\eta\left(t, \gamma_{0}\left(s_{1}, s_{2}, \ldots, s_{l}\right)\right) \|_{\lambda} \leq \rho_{0} .\right.
$$


We claim that for large $T$,

$$
\max _{\left(s_{1}, s_{2}, \ldots, s_{l}\right) \in\left[\frac{1}{R^{2}}, 1\right]^{l}} \Phi_{\lambda}\left(\eta\left(T, \gamma_{0}\left(s_{1}, s_{2}, \ldots, s_{l}\right)(x)\right)\right)<\max \left\{m_{0}, c_{\Gamma}-\frac{1}{2} \tau_{0} \mu\right\},
$$

where $m_{0}$ is given in (4.12), $\tau_{0}=\max \left\{\sigma_{0}, \sigma_{0} / \rho_{0}\right\}$.

In fact, if $\gamma_{0}\left(s_{1}, s_{2}, \ldots, s_{l}\right)(x) \notin A_{\mu}^{\lambda}$, then by (4.13), we have $\Phi_{\lambda}\left(\eta\left(T, \gamma_{0}\left(s_{1}, s_{2}\right.\right.\right.$, $\left.\left.\left.\ldots, s_{l}\right)(x)\right)\right) \leq m_{0}$ and thus (4.14) holds. If $\gamma_{0}\left(s_{1}, s_{2}, \ldots, s_{l}\right)(x) \in A_{\mu}^{\lambda}$, we need to study the behavior of $\tilde{\eta}(t)=\eta\left(t, \gamma_{0}\left(s_{1}, s_{2}, \ldots, s_{l}\right)\right.$. Setting $\tilde{d}_{\lambda}:=\min \left\{d_{\lambda}, \sigma_{0}\right\}$ and $T=\sigma_{0} \mu /\left(2 d_{\lambda}\right)$. Now we distinguish two cases:

(1) $\tilde{\eta}(t) \in A_{3 \mu / 2}^{\lambda}$ for all $t \in[0, T]$.

(2) $\tilde{\eta}\left(t_{0}\right) \in \partial A_{3 \mu / 2}^{\lambda}$ for some $t_{0} \in[0, T]$.

If (1) holds, we have $\Psi(\tilde{\eta}(t)) \equiv 1$ and $\left\|\Phi_{\lambda}^{\prime}(\tilde{\eta}(t))\right\|_{\lambda} \geq \tilde{d}_{\lambda}$ for all $t \in[0, T]$. Thus, by (4.9), we have

$$
\begin{aligned}
\Phi_{\lambda}(\tilde{\eta}(T)) & =\Phi_{\lambda}\left(\gamma_{0}\left(s_{1}, s_{2}, \ldots, s_{l}\right)\right)+\int_{0}^{T} \frac{d}{d s} \Phi_{\lambda}(\tilde{\eta}(t)) d s \\
& =\Phi_{\lambda}\left(\gamma_{0}\left(s_{1}, s_{2}, \ldots, s_{l}\right)\right)-\int_{0}^{T} \Psi(\tilde{\eta}(s))\left\|\Phi_{\lambda}^{\prime}(\tilde{\eta}(s))\right\|_{\lambda} d s \\
& \leq c_{\Gamma}-\int_{0}^{T} \tilde{d}_{\lambda} d s \\
& =c_{\Gamma}-\tilde{d}_{\lambda} T \\
& =c_{\Gamma}-\frac{1}{2} \sigma_{0} \mu \leq c_{\Gamma}-\frac{1}{2} \tau_{0} \mu .
\end{aligned}
$$

If (2) holds, there exists $0 \leq t_{1} \leq t_{1} \leq T$ such that

$$
\begin{aligned}
& \tilde{\eta}\left(t_{1}\right) \in \partial A_{u}^{\lambda}, \\
& \tilde{\eta}\left(t_{2}\right) \in \partial A_{3 \mu / 2}^{\lambda}, \\
& \tilde{\eta}(t) \in A_{3 \mu / 2}^{\lambda} \backslash A_{u}^{\lambda}, \quad \text { for all } t \in\left[t_{1}, t_{2}\right] .
\end{aligned}
$$

It follows from (4.17)

$$
\left\|\tilde{\eta}\left(t_{2}\right)\right\|_{\lambda, \mathbb{R}^{2} \backslash \Omega_{\Gamma}^{\prime}}=\frac{3 \mu}{2}
$$

or

$$
\left|\Phi_{\lambda, \Omega_{j_{0}}^{\prime}}\left(\tilde{\eta}\left(t_{2}\right)\right)-c_{j_{0}}\right|=\frac{3 \mu}{2} .
$$

for some $j_{0} \in \Gamma$.

Now we consider the latter case, the former case can be obtained in a similar way. By (4.16),

$$
\left|\Phi_{\lambda, \Omega_{j_{0}}^{\prime}}\left(\tilde{\eta}\left(t_{1}\right)\right)-c_{j_{0}}\right| \leq \mu
$$


thus, we obtain

$$
\begin{aligned}
& \left|\Phi_{\lambda, \Omega_{j_{0}}^{\prime}}\left(\tilde{\eta}\left(t_{2}\right)\right)-\Phi_{\lambda, \Omega_{j_{0}}^{\prime}}\left(\tilde{\eta}\left(t_{1}\right)\right)\right| \\
& \quad \geq\left|\Phi_{\lambda, \Omega_{j_{0}}^{\prime}}\left(\tilde{\eta}\left(t_{2}\right)\right)-c_{j_{0}}\right|-\left|\Phi_{\lambda, \Omega_{j_{0}}^{\prime}}\left(\tilde{\eta}\left(t_{1}\right)\right)-c_{j_{0}}\right| \\
& \quad \geq \frac{1}{2} \mu .
\end{aligned}
$$

On the other hand, by the mean value theorem, there exists $t_{3} \in\left(t_{1}, t_{2}\right)$ such that

$$
\left|\Phi_{\lambda, \Omega_{j_{0}}^{\prime}}\left(\tilde{\eta}\left(t_{2}\right)\right)-\Phi_{\lambda, \Omega_{j_{0}}^{\prime}}\left(\tilde{\eta}\left(t_{1}\right)\right)\right|=\left|\Phi_{\lambda, \Omega_{j_{0}}^{\prime}} \cdot \frac{d \tilde{\eta}}{d t}\left(t_{3}\right)\right|\left(t_{2}-t_{1}\right)
$$

Moreover, from (4.10) and (4.14), we have

$$
t_{2}-t_{1} \geq \frac{\mu}{2 \rho_{0}} .
$$

Thus, one has

$$
\begin{aligned}
\Phi_{\lambda}(\tilde{\eta}(T)) & =\Phi_{\lambda}\left(\gamma_{0}\left(s_{1}, s_{2}, \ldots, s_{l}\right)\right)+\int_{0}^{T} \frac{d}{d s} \Phi_{\lambda}(\tilde{\eta}(t)) d s \\
& =\Phi_{\lambda}\left(\gamma_{0}\left(s_{1}, s_{2}, \ldots, s_{l}\right)\right)-\int_{0}^{T} \Psi(\tilde{\eta}(s))\left\|\Phi_{\lambda}^{\prime}(\tilde{\eta}(s))\right\|_{\lambda} d s \\
& \leq c_{\Gamma}-\int_{t_{1}}^{t_{2}} \Psi(\tilde{\eta}(s))\left\|\Phi_{\lambda}^{\prime}(\tilde{\eta}(s))\right\|_{\lambda} d s \\
& =c_{\Gamma}-\sigma_{0}\left(t_{2}-t_{1}\right) \\
& =c_{\Gamma}-\frac{1}{2} \sigma_{0} \mu \leq c_{\Gamma}-\frac{1}{2} \tau_{0} \mu .
\end{aligned}
$$

and so (4.15) is proved. Now we recall that $\tilde{\eta}(T)=\eta\left(T, \gamma_{0}\left(s_{1}, s_{2}, \ldots, s_{l}\right)\right) \in \Lambda_{*}$, thus

$$
b_{\lambda, \Gamma} \leq \Phi_{\lambda}(\tilde{\eta}(T)) \leq \max \left\{m_{0}, c_{\Gamma}-\frac{1}{2} \tau_{0} \mu\right\} .
$$

But by Corollary 4.4, we know $b_{\lambda, \Gamma} \rightarrow c_{\Gamma}$ as $\lambda \rightarrow \infty$, this is a contradiction to (4.18), it shows that $\Phi_{\lambda}(u)$ has a critical point $u \in A_{u}^{\lambda}$ for large $\lambda$ and we have completed the proof of the proposition.

Proof of Theorem 1.1. From Proposition 4.6, there exists a family of nontrivial solutions $\left(u_{\lambda}\right)$ to problem (3.5) verifying the following properties.

(i) For fixed $\mu>0$, there exists $\lambda^{*}$ such that

$$
\left\|u_{\lambda}\right\|_{\lambda, \mathbb{R}^{2} \backslash \Omega_{\Gamma}^{\prime}} \leq \mu, \quad \forall \lambda \geq \lambda^{*} .
$$

Thus, from proof of Proposition 3.6, $\mu$ fixed sufficiently small, we can conclude that

$$
\left\|\left|u_{\lambda}\right|\right\|_{\infty, \mathbb{R}^{2} \backslash \Omega_{\Gamma}^{\prime}}^{2} \leq a, \quad \forall \lambda \geq \lambda^{*},
$$


which shows that $u_{\lambda}$ is a nontrivial solution to problem (1.10).

(ii) Fixing $\lambda_{n} \rightarrow \infty$ and $\mu_{n} \rightarrow 0$, the sequence $\left\{u_{\lambda_{n}}\right\}$ verifies

$$
\begin{aligned}
& \Phi_{\lambda_{n}}^{\prime}\left(u_{\lambda_{n}}\right)=0 \forall n \in N, \\
& \left\|u_{\lambda_{n}}\right\|_{\lambda_{n}, \mathbb{R}^{2} \backslash \Omega_{\Gamma}^{\prime}} \rightarrow 0, \\
& \Phi_{\lambda_{n}, j}^{\prime}\left(u_{\lambda_{n}}\right) \rightarrow c_{j} \quad \forall j \in \Gamma .
\end{aligned}
$$

Thus, from proposition 3.2, we have

$$
u_{\lambda_{n}} \rightarrow u \text { in } H_{A}^{1}\left(\mathbb{R}^{2}\right) \text { with } u \in H_{A}^{0,1}\left(\Omega_{\Gamma}\right),
$$

from which the proof of Theorem 1.1 follows.

Acknowledgements C. Ji was supported by Shanghai Natural Science Foundation (18ZR1409100).

Open Access This article is licensed under a Creative Commons Attribution 4.0 International License, which permits use, sharing, adaptation, distribution and reproduction in any medium or format, as long as you give appropriate credit to the original author(s) and the source, provide a link to the Creative Commons licence, and indicate if changes were made. The images or other third party material in this article are included in the article's Creative Commons licence, unless indicated otherwise in a credit line to the material. If material is not included in the article's Creative Commons licence and your intended use is not permitted by statutory regulation or exceeds the permitted use, you will need to obtain permission directly from the copyright holder. To view a copy of this licence, visit http://creativecommons.org/ licenses/by/4.0/.

\section{References}

[1] Alves, C.O.: Existence of multi-bump solutions for a class of quasilinear problems. Adv. Nonlinear Stud. 6, 491-509 (2006)

[2] Alves, C.O., De Morais Filho, D.C., Souto, M.A.S.: Multiplicity of positive solutions for a class of problems with critical growth in $\mathbb{R}^{N}$. Proc. Edinb. Math. Soc. 52, 1-21 (2009)

[3] Alves, C.O., Figueiredo, G.M., Furtado, M.F.: Multiple solutions for a nonlinear Schrödinger equation with magnetic fields. Comm. Partial Differ. Equ. 36, 1565-1586 (2011)

[4] Alves, C.O., Ji, C.: Multi-bump positive solutions for a logarithmic Schrödinger equation with deepening potential well. arXiv:1908.09153 [math.AP]

[5] Alves, C.O., Miyagaki, O.H., Souto, M.A.S.: Multi-bump solutions for a class of quasilinear equations on $\mathbb{R}$. Commun. Pure Appl. Anal. 11, 829-844 (2012)

[6] Alves, C.O., Nóbrega, A.B., Yang, M.B.: Multi-bump solutions for Choquard equation with deepning potential well. Calc. Var. Partial Differ. Equ. 55 art. 48, 28 pp (2016)

[7] Alves, C.O., Pereira, D.S.: Multiplicity of multi-bump type nodal solutions for a class of elliptic problems with exponential critical growth in $\mathbb{R}^{2}$. Proc. Edinb. Math. Soc. 60, 273-297 (2017)

[8] Alves, C.O., Souto, M.A.S.: Multiplicity of positive solutions for a class of problems with exponentical critical growth in $\mathbb{R}^{2}$. J. Differ. Equ. 244, 1502-1520 (2008) 
[9] Alves, C.O., Yang, M.B.: Existence of positive multi-bump solutions for a SchrödingerPoisson system in $\mathbb{R}^{3}$. Discrete Contin. Dyn. Syst. 36, 5881-5910 (2016)

[10] Bahrouni, A., Ounaies, H., Rădulescu, V.D.: Infinitely many solutions for a class of sublinear Schrödinger equations with indefinite potentials. Proc. R. Soc. Edinb. Sect. A 145(3), 445-465 (2015)

[11] Bahrouni, A., Ounaies, H., Rădulescu, V.D.: Bound state solutions of sublinear Schrödinger equations with lack of compactness. Rev. R. Acad. Cienc. Exactas Fís. Nat. Ser. A Mat. RACSAM 113(2), 1191-1210 (2019)

[12] Bartsch, T., Pankov, A., Wang, Z.Q.: Nonlinear Schrödinger equations with steep potential well. Commun. Contemp. Math. 3, 549-569 (2001)

[13] Barile, S., Figueiredo, G.M.: An existence result for Schrödinger equations with magnetic fields and exponential critical growth. J. Elliptic Parabol. Equ. 3, 105-125 (2017)

[14] Berestycki, H., Lions, P.L.: Nonlinear scalar field equations, I existence of a ground state. Arch. Rational Mech. Anal. 82, 313-345 (1983)

[15] Cao, D.M.: Nonlinear solutions of semilinear elliptic equations with critical exponent in $\mathbb{R}^{2}$. Commun. Partial Differ. Equ. 17, 407-435 (1992)

[16] Chabrowski, J., Szulkin, A.: On the Schrödinger equation involving a critical Sobolev exponent and magnetic field. Topol. Methods Nonlinear Anal. 25, 3-21 (2005)

[17] Cingolani, S., Secchi, S.: Semiclassical limit for nonlinear Schördinger equation with electromagnetic fields. J. Math. Anal. Appl. 275, 108-130 (2002)

[18] d'Avenia, P., Ji, C.: Multiplicity and concentration results for a magnetic Schrödinger equation with exponential critical growth in $\mathbb{R}^{2}$. Int. Math. Res. Not. (2020). https:// doi.org/10.1093/imrn/rnaa074

[19] del Pino, M., Felmer, P.L.: Local mountain passes for semilinear elliptic problems in unbounded domains. Calc. Var. Partial Differ. Equ. 4, 121-137 (1996)

[20] Díaz, J.I.: On the ambiguous treatment of the Schrödinger equation for the infinite potential well and an alternative via singular potentials: the multi-dimensional case SeMA J. (2017). https://doi.org/10.1007/s40324-017-0115-3

[21] Ding, Y.H., Tanaka, K.: Multiplicity of positives solutions of a nonlinear Schrödinger equations. Manuscr. Math. 112, 109-135 (2003)

[22] Ding, Y.H., Wang, Z.Q.: Bound states of nonlinear Schrödinger equations with magnetic fields. Annali Mat. Pura Appl. 190, 427-451 (2011)

[23] Esteban, M., Lions, P.L.: Stationary solutions of nonlinear Schrödinger equations with an external magnetic field. PDE Calc. Var. 1, 401-499 (1989)

[24] Floer, A., Weinstein, A.: Nonspreading wave packets for the cubic Schrödinger equation with a bounded potential. J. Funct. Anal. 69, 397-408 (1986)

[25] Gamow, G.: Zur Quantentheorie des Atomkernes. Zeitschrift für Physik 51(204), 204$212(1928)$

[26] Gilbarg, D., Trudinger, N.: Elliptic Partial Differential Equations of Second Order. Springer, New York (1983)

[27] Guo, Y.X., Tang, Z.W.: Multi-bump bound state solutions for the quasilinear Schrödinger equation with critical frequency. Pac. J. Math. 270, 49-77 (2014)

[28] Han, P.: Solutions for singular critical growth Schrödinger equation with magnetic field. Port. Math. (N.S.) 63, 37-45 (2006)

[29] Joglekar, Y.N.: Particle in a box with $a$-function potential: strong and weak coupling limits. Am. J. Phys. 77, 734-736 (2009)

[30] Kato, T.: Remarks on holomorphic families of Schrödinger and Dirac operators, in Differential equations. In: Knowles, I., Lewis, R. (eds.) North-Holland Mathematics Studies, vol. 92, pp. 341-352. North-Holland, Amsterdam (1984)

[31] Kurata, K.: Existence and semi-classical limit of the least energy solution to a nonlinear Schrödinger equation with electromagnetic fields. Nonlinear Anal. 41, 763-778 (2000) 
[32] Lieb, E.H., Loss, M.: Analysis, 2nd Edition, Graduate Studies in Mathematics. American Mathematical Society, Providence (2001)

[33] Liang, S.H., Shi, S.Y.: On multi-bump solutions of nonlinear Schrödinger equation with electromagnetic fields and critical nonlinearity in $\mathbb{R}^{N}$. Calc. Var. Partial Differ. Equ. 56 art. 25, 29pp (2017)

[34] Liang, S.H., Zhang, J.H.: Multi-bump solutions for a class of Kirchhoff type problems with critical growth in $\mathbb{R}^{N}$. Topol. Methods Nonlinear Anal. 48, 71-101 (2016)

[35] Mingqi, X., Rădulescu, V.D., Zhang, B.: A critical fractional Choquard-Kirchhoff problem with magnetic field. Commun. Contemp. Math. 21(4), 1850004, 36pp (2019)

[36] Mott, N.F.: An Outline of Wave Mechanics. Cambridge University Press, Cambridge (1930)

[37] Oh, Y.G.: Existence of semi-classical bound states of nonlinear Schrödinger equations with potentials of the class. Commun. Partial Differ. Equ. 13, 1499-1519 (1988)

[38] Papageorgiou, N.S., Rădulescu, V.D., Repovš, D.D.: Nonlinear Analysis: Theory and Methods. Springer Monographs in Mathematics. Springer, Cham (2019)

[39] Tang, Z.W.: Multi-bump bound states of nonlinear Schrödinger equations with electromagnetic fields and critical frequency. J. Differ. Equ. 245, 2723-2748 (2008)

[40] Willem, M.: Minimax Theorems. Birkhäuser, Boston (1996)

Publisher's Note Springer Nature remains neutral with regard to jurisdictional claims in published maps and institutional affiliations. 